\title{
Lrrce1 and Ccdc61 are conserved effectors of multiciliated cell function
}

Aude Nommick ${ }^{1}$, Camille Boutin ${ }^{1}$, Olivier Rosnet ${ }^{1}$, Elsa Bazellières ${ }^{1}$, Virginie Thomé ${ }^{1}$,

Etienne Loiseau ${ }^{2}$, Annie Viallat ${ }^{2}$, Laurent Kodjabachian, ${ }^{1, *}$

1. Aix Marseille Univ, CNRS, IBDM, Marseille, France

2. Aix Marseille Univ, CNRS, CINaM, Marseille, France

* corresponding author: laurent.kodjabachian@univ-amu.fr

Running title: Lrrcc1 and Ccdc61 in vertebrate multiciliated cells 


\begin{abstract}
Ciliated epithelia perform a variety of essential functions across animal evolution, ranging from locomotion of marine organisms to mucociliary clearance of airways in mammals. These epithelia are composed of multiciliated cells (MCCs) harbouring myriads of motile cilia, which rest on modified centrioles called basal bodies (BBs), and beat coordinately to generate directed fluid flows. Thus, BB biogenesis and organization is central to MCC function. In basal eukaryotes, the coiled-coil domain proteins Lrrcc1 and Ccdc61 were shown to be required for proper BB construction and function. Here, we used the Xenopus embryonic ciliated epidermis to characterize Lrrce1 and Ccdc61 in vertebrate MCCs. We found that they both encode BB components, with a prominent association to striated rootlets. Knocking down either gene caused defects in BB docking, spacing, and polarization. Moreover, their depletion impaired the apical cytoskeleton, and altered ciliary beating. Consequently, cilia-powered fluid flow was greatly reduced in morphant tadpoles, which displayed enhanced mortality when exposed to pathogenic bacteria. This work illustrates how integration across organizational scales make elementary BB components essential for the emergence of the physiological function of ciliated epithelia.
\end{abstract}

Keywords: Xenopus, multiciliated cell, Lrrcc1, Ccdc61, centriole, basal body, basal foot, striated rootlet, polarity, apical cytoskeleton, ciliary beating 


\section{Introduction}

Multiciliated epithelia are composed of multiciliated cells (MCCs) harboring numerous motile cilia. Ciliary beating generates powerful strokes essential for a variety of physiological functions in animals (Meunier and Azimzadeh, 2016). In aquatic organisms of the Lophotrochozoan and Echinodermata phyla, coordinated MCC beating is required for locomotion, clearance and transport of particles, and for feeding of larvae. In vertebrates, MCCs produce external or internal fluid flows. In lungfish, the ciliated epidermis clears the animal of particles and settling organisms before hatching (Kemp, 1996). In amphibian embryos, several roles have been proposed for the ciliated epidermis: prevention of micro-organisms and debris from attaching to the epidermis, pre-hatching rotation and post-hatching gliding, respiratory gas exchange, movement of surface mucus films and transportation of chemical signals to the olfactory organs (Nokhbatolfoghahai et al., 2006). Which of those roles is carried out by MCCs of the Xenopus embryonic epidermis remains unclear, despite a recent wealth of mechanistic studies in this model (Boutin and Kodjabachian, 2019). In mammals, among other functions, MCCs help circulation of the cerebrospinal fluid in the central nervous system, mucociliary clearance of pathogens and pollutants from airways, and transportation of gametes in genital tracts (Spassky and Meunier, 2017). Consequently, mutations in genes necessary for multiple cilia formation or beating cause familial syndromes characterized by severe chronic airway infections, and an elevated risk of infertility (Spassky and Meunier, 2017). A precise multiscale organization of ciliary beating is required to establish a robust and directed flow at the surface of ciliated epithelia. At the cellular scale, all cilia must beat in the same direction, and at the tissue scale the beating direction must be coordinated between neighboring cells. The Planar Cell Polarity pathway plays a prominent role in the establishment and maintenance of cilia polarity, both at tissue and cellular scales (Boutin et al., 2014; Chien et al., 2015; Mitchell et al., 2009; Park et al., 2008; Walentek et al., 2017). 
Motile cilia of MCCs are built via a multistep process (Boutin and Kodjabachian, 2019; Spassky and Meunier, 2017). First, numerous centrioles must be produced and subsequently released in the cytoplasm. Next, centrioles migrate and dock at the apical surface, where they acquire a regular distribution and a coordinated orientation. Finally, ciliary axonemes extend from docked centrioles, and metachronal waves of ciliary beating are initiated and subsequently reinforced by mechanical feedback from the flow, which refines the coordination of cilia polarity. During their journey towards the surface, centrioles mature into basal bodies (BBs) by acquiring basal foot (BF) and rootlet appendages, which localize asymmetrically and are essential for cilia polarization (Meunier and Azimzadeh, 2016). In Xenopus epidermal MCCs, two different types of rootlets attach to the proximal end of BBs. The most prominent rootlet has a fan shape and is localized opposite to the BF, which itself is positioned distally on the BB. The second rootlet is much longer and thinner and dives into the cytoplasm (Zhang and Mitchell, 2015). These appendages confer an intrinsic polarity to BBs, which in mature MCCs reflects the direction of ciliary beating, with the BF pointing in the direction of the effective stroke.

MCC cilia formation and organization relies on close interactions between BBs and cytoskeletal elements (Boutin and Kodjabachian, 2019; Meunier and Azimzadeh, 2016). The transport and docking of centrioles/BBs to the apical surface is dependent on acto-myosin-based mechanisms (Boisvieux-Ulrich et al., 1990; Epting et al., 2015; Kulkarni et al., 2018; Lemullois et al., 1988; Miyatake et al., 2015; Park et al., 2008). Once docked, neighboring BBs are linked by subapical actin filaments and apical microtubules (MTs) emanating from rootlets and BFs, respectively. The geometrical network hence made ensures regular BB spacing and coordinated orientation over the apical cell surface (Antoniades et al., 2014; Lemullois et al., 1988; Park et al., 2006; Werner et al., 2011; Yasunaga et al., 2015). Chemical interference with actin or MT networks leads to BB disorganization and impaired ciliary function (Herawati et al., 2016; Werner et al., 
2011). Reciprocally, depletion of specific centriolar components, which prevents the formation of appendages and/or preclude BB/cytoskeleton interactions, alter BB organization and ciliary function (Antoniades et al., 2014; Bustamante-Marin et al., 2019; Clare et al., 2014; Herawati et al., 2016; Kulkarni et al., 2018; Kunimoto et al., 2012; Turk et al., 2015; Walentek et al., 2016).

Lrrcc1 (Leucine rich repeat coiled-coil domain containing 1) and Ccdc61 (Coiled-coil domaincontaining protein 61) are structural proteins, conserved from Chlamydomonas to human, which are involved in centriole appendage biogenesis and function, both at the centrosome and at ciliary BBs (Adams et al., 1985; Barenz et al., 2018; Basquin et al., 2019; Bengueddach et al., 2017; Hoops et al., 1984; Muto et al., 2008; Ochi et al., 2020; Pizon et al., 2020; Silflow et al., 2001; Wright et al., 1983). In MCCs of the planarian epidermis, Lrrcc1 (Vfl1) and Ccdc61 (Vfl3) depletion causes structural BB defects, thus perturbing cilia orientation, and altering the direction of locomotion (Basquin et al., 2019). The importance of Lrrcc1 and Ccdc61 in vertebrate MCCs remains unknown. Here, we used the Xenopus laevis ciliated epidermis as a model to shed light on this issue. We decided to comparatively study Lrrcc1 and Ccdc61, based on their shared biological functions in Chlamydomonas and Schmidtea (Adams et al., 1985; Basquin et al., 2019; Hoops et al., 1984; Wright et al., 1983), and their reported physical interaction in an unbiased human proteomic screen (Hein et al., 2015). In Xenopus, MCCs are specified deeply, in the inner cellular layer of the epidermis before intercalating at regular intervals into the outer cellular layer. BB synthesis is initiated while MCCs are still in the inner epidermal layer; BB docking, distribution, orientation and ciliogenesis are completed when MCCs have radially intercalated and expanded their apical surface.

We report here that Lrrcc1 and Ccdc61 are both associated to Xenopus MCC centrioles, with a preferential localization at rootlets of mature BBs. We found that knocking down either gene impacts ciliated epithelium biogenesis at multiple scales. At the organelle scale, Ccdc61 is 
required for rootlet association of Pericentrin (Pcnt), that we characterize as a novel marker of this appendage in Xenopus BBs. At the cellular scale, we show that Lrrcc1 and Ccdc61 are required for proper organization of BBs. In addition, MCCs depleted for either gene present important defects in apical cytoskeleton organization. Finally, at the scale of the embryo, Lrrcc1 and Ccdc61 knock-down revealed their importance for the generation of a directed fluid flow and the resistance to opportunistic pathogens. This study bridges multiple scales of analysis to reveal how intracellular disorganization of MCCs can impair the physiology of the whole organism.

\section{Results}

\section{Lrrer1 and Cedc61 are associated to centrioles and basal bodies in MCCs}

Single cell RNA sequencing (scRNA-seq) of Xenopus tropicalis embryos revealed that lrrccl and $c c d c 61$ are specifically expressed in ciliated epidermal cells from gastrula stages onwards (Briggs et al., 2018), and both genes were found to be activated by the Multicilin/E2F4/5 complex, which is known to be necessary and sufficient for vertebrate MCC differentiation (Ma et al., 2014). We used whole mount in situ hybridization to analyze the localization of lrrcc 1 and ccdc61 transcripts in Xenopus laevis embryos. At early tailbud stage 20, both genes displayed a "salt and pepper" pattern typical of epidermal MCCs (Fig. S1A). Next, we analyzed protein subcellular localization at different time points during MCC differentiation. First, we used antibody staining to analyze the distribution of the endogenous Lrrcc1 protein. At stage 16, Lrrcc1 was detected in the vicinity of Centrin-positive cytoplasmic clouds, where centriole synthesis occurs (Fig. 1A). At stage 18, when released centrioles start migrating towards the apical surface, Lrrcc1 appeared closely associated to individual neo-centrioles marked by Centrin (Fig. 1B). At stage 31, Lrrcc1 was associated to mature BBs docked at the apical surface. Interestingly, the Lrrcc1 signal was systematically located on one side of Centrin- 
positive BBs. In orthogonal view, the signal flanked the $\mathrm{BB}$ and extended towards the cytoplasm (Fig. 1C). This localization suggested that Lrrcc1 associates to the BB rootlet. To further confirm this, we carefully analyzed the 3D distribution of Lrrcc1 relative to Centrin and $\gamma$-Tubulin, known to localize to the BF cap in MCCs (Clare et al., 2014; Hagiwara et al., 2000). On a top view, Centrin appeared as a single dot (Fig. 1D and Fig. S1B). Two pools of $\gamma$-Tubulin were observed juxtaposed to the BB. The first one appeared as a dot with strong intensity juxtaposed to the BB core. A lateral view revealed that this dot was located at a depth similar to that of the BB (Fig. 1D1, Fig. S1B). This pool corresponds to the $\gamma$-Tubulin previously described at the BF. In contrast, the second pool of $\gamma$-Tubulin displayed a less intense signal. It appeared as one or two dots localized opposite to the BF with respect to the BB (Fig. S1B). This pool localized deeper, extending from the BB into the cytoplasm, in a position compatible with the double rootlets described in Xenopus MCCs (Zhang and Mitchell, 2015). The Lrrcc1 signal appeared either as a single dot (Fig. 1D1), or as double dots (Fig. 1D2), below the Centrin signal and extending towards the cytoplasm. This analysis revealed that the endogenous Lrrcc1 protein localized to the two types of rootlets of Xenopus epidermal MCCs. It also revealed the association of $\gamma$-Tubulin to these appendages.

The lack of cross-reacting antibodies prevented us from analyzing the endogenous Ccdc61 protein in Xenopus MCCs. Therefore, we injected a synthetic transcript coding for a Ccdc61GFP fusion protein to get an insight on the putative distribution of endogenous Ccdc61 during MCC differentiation. At all stages analyzed, Ccdc61-GFP was observed in close vicinity of centrioles (Fig. 1E-G). In mature MCCs, triple staining with Centrin and $\gamma$-Tubulin indicated that Ccdc61-GFP was found opposite to the BF, in a position compatible with the proximal part of the rootlet (Fig. 1H). A similar localization was recently reported for a Ccdc61-RFP fusion in Xenopus epidermal MCCs (Ochi et al., 2020). We conclude that Lrrcc1 and Ccdc61 preferentially associate to rootlets in mature epidermal MCCs of Xenopus. 
Altogether these data suggest that Lrrcc1 and Ccdc61 are associated to epidermal MCC centrioles from the time of their release in the cytoplasm through the phases of docking, BB maturation, ciliary growth and maintenance.

\section{Lrrce1 and Cedc61 depletion impairs centriole docking, spacing and orientation}

The subcellular distribution of Lrrcc1 and Ccdc61 during MCC differentiation suggested that they could be involved in BB apical docking as well as distribution and orientation at the apical surface. To investigate this, we knocked down lrrccl and $c c d c 61$ through injection in the presumptive epidermis of two independent morpholino antisense oligonucleotides (MOs) designed to block translation (MO-ATG) or splicing (MO-Spl) (Fig. S2A). The efficiency of MOs was established through the reduction of immunofluorescent signal intensity of endogenous Lrrcc1, or co-injected Ccdc61-GFP (Fig. S2B, C). We performed immunostaining at stage 31 to analyze BB organization. As previously described (Werner et al., 2011), BBs displayed a stereotypical organization in mature control MCCs. They were all docked, quite evenly distributed at the apical surface and oriented in a coordinated manner (Fig. 2A, C, E). In contrast, Lrrcc1 and Ccdc61 knock-down drastically impaired centriole organization and morphant cells could be classified in two phenotypic categories. The first phenotypic class was characterized by clusters of centrioles stuck in the upper half of the cytoplasm (Fig. 2A, Fig. S3A). To quantify this defect, we analyzed the apico-basal (A-B) distribution of centrioles along the Z-axis of confocal stack acquisitions. In control cells, most centrioles localized within the first 1,6 $\mu \mathrm{m}$ below the apical cell surface (Fig. 2B). In contrast, in Lrrcc1-ATG and Ccdc61ATG morphant cells most centrioles localized deeper, between 1,6 $\mu \mathrm{m}$ and $5.6 \mu \mathrm{m}$ from the surface (Fig. 2B). Similar results were obtained with Lrrcc1-Spl and Ccdc61-Spl MOs (Fig. $\mathrm{S} 3 \mathrm{~B})$. In the second phenotypic class, most centrioles properly localized at the apical surface, but displayed irregular spacing and a randomized orientation (Fig. 2C, E and Fig. S3C, E). To 
quantify BB spacing defects, we applied Delaunay triangulation between the centroids of docked centrioles and measured the area of the obtained triangles. In the control situation, the even distribution of BBs resulted in many triangles of similar area that tightly distributed around the median (Fig. 2C, D and Fig. S3C, D). In contrast, in morphant cells, variable distances between BBs resulted in a broader distribution of triangles around the median (Fig. 2C, D and Fig. S3C, D). We revealed BB orientation by immunostaining the BB core (Centrin) and the offset BF (intense $\gamma$-Tubulin spot), which allowed us to automatically extract orientation vectors and plot their circular distribution. In control cells, vector angles tightly distributed around the mean, indicating that BBs oriented in the same direction. In Lrrcc1 and Ccde61 morphant cells, the intense $\gamma$-Tubulin signal was largely preserved, suggesting that the integrity of the BF was maintained. However, vector angles were widely distributed around the mean, indicating a randomization of $\mathrm{BB}$ orientation (Fig. 2E and Fig. S3E). Accordingly, circular standard deviation values were significantly higher in morphants, as compared to control (Fig. 2F and Fig. S3F). The Rayleigh statistical test also revealed a higher percentage of morphant MCCs, in which no significant mean vector could be defined, as compared to control (Fig. 2G and Fig. $\mathrm{S} 3 \mathrm{G})$.

In both Lrrcc1 and Ccdc61 morphant MCCs, cilia could be observed suggesting that those proteins are not required for ciliary growth per se (Fig. S4). Importantly, for both genes and both types of MOs, docking, spacing and orientation phenotypes were rescued by co-injection of lrrccl or $c c d c 61$ mRNA constructs lacking (lrrcc1), or silently mutated (ccdc61) on the MObinding sequence (Fig. 2B, D, F, Fig. S3B, D, F and S5).

Altogether, these data show that Lrrcc1 and Ccdc61 are required for correct apical migration and/or docking of BBs, as well as for their proper planar distribution and orientation at the apical surface. 


\section{Cedc61 is required for Pericentrin association to rootlet}

Our localization and functional data suggested that Lrrce1 and Ccdc61 could participate to the formation of rootlet appendages in Xenopus MCCs. To analyze this possibility, we performed TEM experiments on stage 31 MCCs from control and morphant embryos. The presence of centriole docking defects was used as a way to ascertain the morphant character of scored MCCs (Fig. 3B). Both fan-shape and thin rootlets could be observed in similar proportions in Lrrcc1 and Ccdc61 morphant cells, as compared to control (Fig. 3A, C). Whenever visible, rootlets did not present obvious structural or positioning defects.

To look for potential molecular defects, we searched for an endogenous rootlet marker to be used in fluorescent imaging. Using home-made antibodies (Fig. S6A, B), we identified Xenopus Pcnt as a bona fide rootlet component. In stage 31 control cells, Pcnt immunostaining presented a dotted pattern, and was associated with Centrin and $\gamma$-Tubulin at the apical surface (Fig. 3D). Analysis of the relative distribution of these proteins in individual BBs revealed that Pcnt was located opposite to the strong BF-associated dot of $\gamma$-Tubulin, in a plane below Centrin. It was present as one or two dots emerging from the BB and extending towards the cytoplasm (Fig 3E, S6C). This analysis revealed that in Xenopus epidermal MCCs, Pcnt specifically localizes at rootlets.

Next, we analyzed Pcnt signal in Lrrce1 and Ccd61 morphant embryos. To avoid immunostaining variability between different embryos, we compared Pcnt signal intensity within mosaic embryos. For Lrrcc1, no differences were observed between non-injected and morphant cells, which, however, clearly displayed randomized BB polarity (Fig. 3G-H). In contrast, a marked decrease of Pcnt signal was observed in Ccdc61 morphant cells, as compared to non-injected cells from the same embryos (Fig. 3G-H). 
Altogether, these experiments suggest that Lrrcc1 and Ccdc61 are not required for building rootlet appendages in Xenopus MCCs. However, Ccdc61 is required for association of the protein Pcnt to the rootlet, which in turn could affect rootlet function and BB organization.

\section{Lrrce1 and Cedc61 depletion disturbs apical cytoskeleton organization}

BB organizational defects observed in Ccdc61 and Lrrcc1 morphant cells could be linked to defective apical cytoskeleton. To test this idea, we first analyzed F-actin networks in MCCs at stage 31 . In control cells, we observed the stereotypical organization in apical and subapical networks that was previously described for mature MCCs (Werner et al., 2011). The apical actin network was organized like a grid surrounding each centriole, and the subapical network located just below was composed of short actin fibers that are known to connect neighboring BBs via their rootlets (Fig. 4A, A'). Both Lrrcc1 and Ccdc61 knockdown caused a global decrease of F-actin staining (Fig. 4D, E). When looking at individualized centrioles, a strong reduction of both apical and subapical F-actin was observed (Fig. 4B-C'). From these results, we conclude that Lrrcc1 and Ccdc61 are, either directly or indirectly, involved in the assembly of the apical filamentous actin networks.

Next, we analyzed the apical MT network in mature MCCs. At stage 31 , anti- $\alpha$-Tubulin antibodies mainly revealed cilia, precluding the analysis of intracellular MT networks in mature MCCs (Fig. S7B). To circumvent this limitation, we adopted a deciliation strategy to deplete cilia-associated signals and visualize intracellular MTs (Fig. S7B). At stage 31, control cells were characterized by a highly organized apical MT network connecting BBs together (Fig. 5A, A'), similar to what has been reported by another method (Werner et al., 2011). In Lrrec1 and Ccdc61 depleted-MCCs, intense $\alpha$-Tubulin signals were found associated to clustered centrioles (Fig. 5B, B', D, D'). When looking at individualized BBs, MTs were clearly visible but the size and spatial organization of filaments appeared very heterogeneous, as compared to 
control (Fig 5C, C', E, E'). These results suggest that Lrrcc1 and Ccdc61 are not necessary for apical MT network polymerization per se, but could have a role, either directly or indirectly, on its organization.

Finally, we analyzed the apical intermediate filament (IF) network using the anti-cytokeratin C-11 antibody. At stage 18, we did not detect IFs inside or at the MCC surface, suggesting that they are not involved in centriole apical migration (Fig. 6A). At stage 31, IFs were organized into a dense grid surrounding each BB (Fig. 6B, B'), similar to what has been described in tracheal MCCs (Tateishi et al., 2017). Strikingly, IF organization was drastically affected in Lrrcc1 and Ccdc61 morphant cells. Overall, the lattice appeared much less dense and the annular organization of IF around BBs was lost (Fig. 6C, C', D, D'). This analysis suggests that Lrrcc1 and Ccdc61 are, directly or indirectly, involved in the establishment of the tight IF network in which BBs are embedded.

Altogether these analyses reveal that Lrrcc1 and Ccdc61 depletion has profound impacts on MCC apical cytoskeleton organization.

\section{Lrrec1 and Cedc61 depletion reduces ciliary beating, impairs flow circulation and increases sensitivity to pathogen}

The perturbed organization of BBs in morphant MCCs is expected to disturb the function of associated cilia, thereby affecting the production of fluid flow at the surface of the embryo. To address this issue, we first analyzed ciliary beating frequency by high-speed video-recording. As previously described, control MCCs performed synchronised and large amplitude effective and recovery strokes, characterized by the ability of cilia to extensively bend (Fig. 7A, and videos 1 and 2)(Werner et al., 2011). In Lrrcc1 and Ccdc61 morphant embryos, we observed three levels of beating defaults: (i) low-amplitude, uncoordinated and disoriented beating, causing occasional collisions between cilia; (ii) cilia performing only small vibrations; (iii) 
extreme cases with totally immobile cilia (Fig. 7A, and videos 1 and 2). Accordingly, the beating frequency, which was about $15 \mathrm{~Hz}$ for the control condition, decreased to lower values in morphant MCCs. Around 20 and $30 \%$ of MCCs were immotile in Lrrce1 and Ccde61 morphant conditions, respectively. In Lrrcc1 morphants, the remaining $80 \%$ of MCCs beat at much slower frequencies than control cells. In contrast, in Ccdc61 morphants, the remaining $70 \%$ of MCCs beat with similar frequencies as controls, suggesting that Ccdc61 depletion may cause an on/off switch (Fig. 7A). Next, we analyzed cilia-generated flow in the surrounding liquid, by live recording of visible dyed microspheres dispersed in the fluid, along the flanks of embryos at stage 31. In control condition, microspheres were moved by a robust flow and traveled the entire length of the embryo in approximately 12 seconds. In contrast, the flow was severely slowed down in Lrrcc1 and Ccdc61 morphant conditions, and the microspheres rarely reached the middle of the embryo after 12 seconds (Fig. 7B, C and video 3).

To address the impact of impaired ciliary flow on the physiology of morphant individuals, we analyzed their susceptibility to pathogen infection. Embryos were incubated for $72 \mathrm{~h}$ with the opportunistic bacteria Aeromonas Hydrophila (Dubaissi et al., 2018), and their survival rate was recorded. Non-injected and GFP-injected embryos were used as controls. For both control conditions, an almost complete survival rate was observed independently of the presence or not of bacteria (Fig. 7D). In absence of bacteria, Lrrcc1 and Ccdc61 morphant embryos displayed a slightly decreased survival rate as compared to control embryos. However, the presence of pathogenic bacteria strongly impacted the survival rate of morphants, starting from $24 \mathrm{~h}$ of incubation. We confirmed by immunostaining that centrioles and cilia were disorganized in morphant embryos from the same experimental series (Fig. S8). This assay suggests that Lrrcc1 and Ccdc61 inactivation leads to a higher susceptibility of embryos to pathogen infection, likely due to reduced cilia-powered clearance. 


\section{Discussion}

In this study, we report a role for Lrrcc1 and Ccdc61 in Xenopus epidermal MCC differentiation and function, extending their evolutionary conserved importance in ciliated cells to vertebrates. At the cellular scale, both Lrrcc1 and Ccdc61 are necessary for the migration and/or docking of BBs at the cell surface and for apical cytoskeleton organization. At a larger scale, both factors are necessary for cilia-powered superficial flow to help survival of the organism, when exposed to environmental pathogens.

Lrrce1 and Ccde61 proteins both contain coiled-coil domains, which are one of the most common structural motifs mediating protein-protein interactions. Search in the IntAct protein interaction database reveals that Lrrcc1 and Ccdc61 physically interact with multiple known factors related to centrosomes, cilia and MT organization or polymerization, among which $30 \%$ are actually shared (Table 1)(Hein et al., 2015). Moreover, 20/26 (Lrrcc1), and 12/23 (Ccdc61) of those interactors were found to be expressed in MCCs of Xenopus tropicalis, as revealed by scRNA-seq (Table 1). Together with their reported interaction, this information is consistent with Lrrcc1 and Ccde61 shared localization and phenotypes, and suggest that these two factors belong to a common molecular network important for BB function in MCCs.

We show that Ccde61 and Lrrcc1 associate to BBs early after their production and maintain this association in fully differentiated MCCs. The early association to neo-synthesized centrioles is compatible with a role in apical migration, which appears to be incomplete in Lrrcc1- and Ccdc61-deficient mature MCCs. Alternatively, apical migration may be unaffected, and defective docking may secondarily cause BBs to dive back into the cytoplasm. The distribution of Lrrcc1 and Ccdc61 at BBs is consistent with their observed requirement for correct MCC organization. This function is shared with Vfl1 (Lrrcc1) and Vfl3 (Ccdc61) orthologs, which are required to organize flagella/cilia in Chlamydomonas, Paramecium and planarians (Adams et al., 1985; Basquin et al., 2019; Bengueddach et al., 2017; Hoops et al., 
1984; Wright et al., 1983). In contrast to studies in these species, however, we did not detect major rootlet structural defects upon Lrrce1 and Ccdc61 depletion, suggesting the existence of redundant mechanisms to build up Xenopus BB appendages.

From their place of birth to their final position at the apical surface, the relocation of centrioles/BBs is intimately linked to cytoskeletal networks (Boisvieux-Ulrich et al., 1990; Herawati et al., 2016; Lemullois et al., 1988; Werner et al., 2011). The organization of these networks evolves during MCC maturation, allowing first to direct BBs towards the apical surface and then to orient and space them. By using chemical cytoskeleton inhibitors, it has been possible to alter migration, orientation or dispersion of BBs, thus attributing specific functions to actin filaments and MTs (Boisvieux-Ulrich et al., 1990; Werner et al., 2011). Our data revealed the primary importance of both Lrrcc1 and Ccdc61 for the establishment of actin filamentous networks. In particular, the absence of sub-apical actin fibers, which connect BBs through their rootlet (Antoniades et al., 2014; Werner et al., 2011), is consistent with the localization of Lrrce1 and Ccdc61 at these sites. In contrast to actin filaments and MTs, the contribution of IFs to the organization of MCCs remains unknown. Our analysis did not reveal the presence of IFs at the stage of centriole apical migration, ruling out their implication at this step. In mature MCCs, a prominent IF network adopts an annular shape around BBs, similar to the apical F-actin network, but which appears to form more basally, extending below the BB level, where rootlets are found (Fig. 4A' and 6B')(Sandoz et al., 1988). This IF network collapsed in MCCs deprived of Lrrcc1 and Ccdc61, suggesting that IFs may stably interact with ciliary rootlets, through molecular complexes containing these two structural proteins. This first rudimentary analysis makes the Xenopus epidermis an attractive paradigm to address the specific role of IFs in MCC organization and function.

In contrast to the BF, which functions as an MT-organizing center (Clare et al., 2014; Kunimoto et al., 2012), the role of the rootlet appendage is less clear. Among proposed functions, it is 
generally believed to serve as an anchor, allowing BBs to maintain their position at the apical surface, and resist mechanical forces generated by ciliary beating (Antoniades et al., 2014; Bustamante-Marin et al., 2019; Yang et al., 2005; Yang and Li, 2005). In line with this, rootlets are scaffolds for molecular interactions, among which ciliary adhesion complexes were found to organize the short actin filaments that cross-link BBs together and help beating synchronization (Antoniades et al., 2014; Walentek et al., 2016; Werner et al., 2011). Here, we observed that Ccdc61, Pcnt and $\gamma$-Tubulin associate to rootlets in mature epidermal MCCs of Xenopus. Ccdc61 was recently shown to be a paralog of the scaffolding protein Sas6, known to establish the 9-fold rotational symmetry of MTs during centriole duplication (Ochi et al., 2020). Interestingly, an evolutionary conserved interaction between Sas6 and Pcnt has recently been reported (Ito et al., 2019). Furthermore, Pcnt is known for its ability to recruit $\gamma$-Tubulin to assemble a macro-molecular complex allowing MT nucleation at the centrosome, and at mitotic spindles (Woodruff et al., 2014). Based on this information and our own observations, it is tempting to propose that Ccdc61 may help Pcnt recruitment/stabilization at the rootlet, which in turn would favour $\gamma$-Tubulin recruitment and MT nucleation. Alternatively, the reported interaction of Ccdc61 with MTs (Ochi et al., 2020) may be important to link pillar MTs emanating from the BF (Clare et al., 2014) with nearby rootlets, which could further strengthen the mechanical coupling between adjacent BBs, to optimize their coordinated orientation and synchronized beating. Future super-resolution and EM studies should investigate the possible link between MTs and rootlets.

Unexpectedly, we found that cilia in Lrrcc1 and Ccdc61 morphant MCCs often beat very poorly. Proper ciliary beating entails correct axonemal structure and delivery of dynein motors to maintain ciliary motion (Huizar et al., 2018; Satir et al., 2014). Although we have not investigated these features, it is possible that the lack of Lrrcc1 and Ccdc61 compromises the 
capacity of rootlets to help trafficking towards the axoneme of essential structural or motor effectors (Gray et al., 2009; Mohan et al., 2013; Park et al., 2008; Yang and Li, 2005).

From the nanoscopic organization of organelles to the cellular function and physiology of the organism, all scales are coupled. The emergence of the locomotor function in planarians is a striking example of such coupling in multiciliated epithelia. In this model, polarization of BBs relies on their chiral construction, which mobilizes Lrrcc1 and Ccd61. This polarization allows the establishment of bilateral symmetry of the ventral multiciliated epidermis, which in turns governs the orientation of the worm movement (Basquin et al., 2019). In addition, retro-control loops exist between the different scales. For instance, in mouse ependymal MCCs, the establishment of a dense actin network that confers stability to BBs and cilia is dependent on active ciliary beating (Mahuzier et al., 2018). Thus, understanding such highly integrated systems implies analyzing multiple scales, as well as their coupling and feedback mechanisms. The present multi-scale study correlates the absence of structural proteins at BB appendages to MCC disorganization, defective flow production and impaired resistance to pathogens. Despite a wealth of studies on the Xenopus ciliated embryonic skin, it was unknown whether ciliapowered flow could limit infections by environmental pathogens. In that respect, the function of the Xenopus mucociliary epidermis is similar to that of the mucociliary epithelium that ensures the clearance of incoming pathogens in mammalian airways. In airways, cilia beating helps to propel a viscous layer of mucus trapping foreign particles, which lies on top of an aqueous periciliary layer. In contrast, in the Xenopus skin, the mucus layer is about $6 \mu \mathrm{m}$ thick and sits right on top of the epithelium (Dubaissi et al., 2018), so that the $15 \mu \mathrm{m}$-long cilia actually beat in water to prevent attachment of micro-organisms. Thus, unlike in airways, the mucus on the frog embryonic skin may not be propelled as a coherent layer, which would explain the much lower density of MCCs necessary in this system. Although additional functions, such as oxygenation of the skin, are not ruled out, our proposed pathogen clearance 
function is consistent with the resorption of MCCs in pre-metamorphic tadpoles (Tasca et al., 2020), at a stage when innate immunity is in place (Robert and Ohta, 2009) .

\section{References}

Adams, G.M., Wright, R.L., Jarvik, J.W., 1985. Defective temporal and spatial control of flagellar assembly in a mutant of Chlamydomonas reinhardtii with variable flagellar number. $\mathrm{J}$ Cell Biol 100, 955-964.

Antoniades, I., Stylianou, P., Skourides, P.A., 2014. Making the connection: ciliary adhesion complexes anchor basal bodies to the actin cytoskeleton. Dev Cell 28, 70-80.

Barenz, F., Kschonsak, Y.T., Meyer, A., Jafarpour, A., Lorenz, H., Hoffmann, I., 2018. Ccdc61 controls centrosomal localization of Cep170 and is required for spindle assembly and symmetry. Mol Biol Cell 29, 3105-3118.

Basquin, C., Ershov, D., Gaudin, N., Vu, H.T., Louis, B., Papon, J.F., Orfila, A.M., Mansour, S., Rink, J.C., Azimzadeh, J., 2019. Emergence of a Bilaterally Symmetric Pattern from Chiral Components in the Planarian Epidermis. Dev Cell 51, 516-525 e515.

Bengueddach, H., Lemullois, M., Aubusson-Fleury, A., Koll, F., 2017. Basal body positioning and anchoring in the multiciliated cell Paramecium tetraurelia: roles of OFD1 and VFL3. Cilia 6,6 .

Boisvieux-Ulrich, E., Laine, M.C., Sandoz, D., 1990. Cytochalasin D inhibits basal body migration and ciliary elongation in quail oviduct epithelium. Cell Tissue Res 259, 443-454.

Boutin, C., Kodjabachian, L., 2019. Biology of multiciliated cells. Curr Opin Genet Dev 56, 17.

Boutin, C., Labedan, P., Dimidschstein, J., Richard, F., Cremer, H., Andre, P., Yang, Y., Montcouquiol, M., Goffinet, A.M., Tissir, F., 2014. A dual role for planar cell polarity genes in ciliated cells. Proc Natl Acad Sci U S A 111, E3129-3138.

Briggs, J.A., Weinreb, C., Wagner, D.E., Megason, S., Peshkin, L., Kirschner, M.W., Klein, A.M., 2018. The dynamics of gene expression in vertebrate embryogenesis at single-cell resolution. Science 360.

Bustamante-Marin, X.M., Yin, W.N., Sears, P.R., Werner, M.E., Brotslaw, E.J., Mitchell, B.J., Jania, C.M., Zeman, K.L., Rogers, T.D., Herring, L.E., Refabert, L., Thomas, L., Amselem, S., Escudier, E., Legendre, M., Grubb, B.R., Knowles, M.R., Zariwala, M.A., Ostrowski, L.E., 2019. Lack of GAS2L2 Causes PCD by Impairing Cilia Orientation and Mucociliary Clearance. Am J Hum Genet 104, 229-245.

Chien, Y.H., Keller, R., Kintner, C., Shook, D.R., 2015. Mechanical strain determines the axis of planar polarity in ciliated epithelia. Curr Biol 25, 2774-2784.

Clare, D.K., Magescas, J., Piolot, T., Dumoux, M., Vesque, C., Pichard, E., Dang, T., Duvauchelle, B., Poirier, F., Delacour, D., 2014. Basal foot MTOC organizes pillar MTs required for coordination of beating cilia. Nat Commun 5, 4888.

Dubaissi, E., Rousseau, K., Hughes, G.W., Ridley, C., Grencis, R.K., Roberts, I.S., Thornton, D.J., 2018. Functional characterization of the mucus barrier on the Xenopus tropicalis skin surface. Proc Natl Acad Sci U S A 115, 726-731. 
Epting, D., Slanchev, K., Boehlke, C., Hoff, S., Loges, N.T., Yasunaga, T., Indorf, L., Nestel, S., Lienkamp, S.S., Omran, H., Kuehn, E.W., Ronneberger, O., Walz, G., Kramer-Zucker, A., 2015. The Rac1 regulator ELMO controls basal body migration and docking in multiciliated cells through interaction with Ezrin. Development 142, 1553.

Gray, R.S., Abitua, P.B., Wlodarczyk, B.J., Szabo-Rogers, H.L., Blanchard, O., Lee, I., Weiss, G.S., Liu, K.J., Marcotte, E.M., Wallingford, J.B., Finnell, R.H., 2009. The planar cell polarity effector Fuz is essential for targeted membrane trafficking, ciliogenesis and mouse embryonic development. Nat Cell Biol 11, 1225-1232.

Hagiwara, H., Kano, A., Aoki, T., Ohwada, N., Takata, K., 2000. Localization of gammatubulin to the basal foot associated with the basal body extending a cilium. Histochem $\mathrm{J} 32$, 669-671.

Hein, M.Y., Hubner, N.C., Poser, I., Cox, J., Nagaraj, N., Toyoda, Y., Gak, I.A., Weisswange, I., Mansfeld, J., Buchholz, F., Hyman, A.A., Mann, M., 2015. A human interactome in three quantitative dimensions organized by stoichiometries and abundances. Cell 163, 712-723.

Herawati, E., Taniguchi, D., Kanoh, H., Tateishi, K., Ishihara, S., Tsukita, S., 2016. Multiciliated cell basal bodies align in stereotypical patterns coordinated by the apical cytoskeleton. J Cell Biol 214, 571-586.

Hoops, H.J., Wright, R.L., Jarvik, J.W., Witman, G.B., 1984. Flagellar waveform and rotational orientation in a Chlamydomonas mutant lacking normal striated fibers. J Cell Biol 98, 818-824.

Huizar, R.L., Lee, C., Boulgakov, A.A., Horani, A., Tu, F., Marcotte, E.M., Brody, S.L., Wallingford, J.B., 2018. A liquid-like organelle at the root of motile ciliopathy. Elife 7.

Ito, D., Zitouni, S., Jana, S.C., Duarte, P., Surkont, J., Carvalho-Santos, Z., Pereira-Leal, J.B., Ferreira, M.G., Bettencourt-Dias, M., 2019. Pericentrin-mediated SAS-6 recruitment promotes centriole assembly. Elife 8.

Kemp, A., 1996. Role of epidermal cilia in development of the Australian lungfish, Neoceratodus forsteri (Osteichthyes: Dipnoi). J Morphol 228, 203-221.

Kulkarni, S.S., Griffin, J.N., Date, P.P., Liem, K.F., Jr., Khokha, M.K., 2018. WDR5 Stabilizes Actin Architecture to Promote Multiciliated Cell Formation. Dev Cell 46, 595-610 e593.

Kunimoto, K., Yamazaki, Y., Nishida, T., Shinohara, K., Ishikawa, H., Hasegawa, T., Okanoue, T., Hamada, H., Noda, T., Tamura, A., Tsukita, S., Tsukita, S., 2012. Coordinated ciliary beating requires Odf2-mediated polarization of basal bodies via basal feet. Cell 148, 189-200.

Lemullois, M., Boisvieux-Ulrich, E., Laine, M.C., Chailley, B., Sandoz, D., 1988. Development and functions of the cytoskeleton during ciliogenesis in metazoa. Biol Cell 63, 195-208.

Ma, L., Quigley, I., Omran, H., Kintner, C., 2014. Multicilin drives centriole biogenesis via E2f proteins. Genes Dev 28, 1461-1471.

Mahuzier, A., Shihavuddin, A., Fournier, C., Lansade, P., Faucourt, M., Menezes, N., Meunier, A., Garfa-Traore, M., Carlier, M.F., Voituriez, R., Genovesio, A., Spassky, N., Delgehyr, N., 2018. Ependymal cilia beating induces an actin network to protect centrioles against shear stress. Nat Commun 9, 2279.

Meunier, A., Azimzadeh, J., 2016. Multiciliated Cells in Animals. Cold Spring Harb Perspect Biol 8.

Mitchell, B., Stubbs, J.L., Huisman, F., Taborek, P., Yu, C., Kintner, C., 2009. The PCP pathway instructs the planar orientation of ciliated cells in the Xenopus larval skin. Curr Biol 19, 924-929. 
Miyatake, K., Kusakabe, M., Takahashi, C., Nishida, E., 2015. ERK7 regulates ciliogenesis by phosphorylating the actin regulator CapZIP in cooperation with Dishevelled. Nat Commun 6, 6666 .

Mohan, S., Timbers, T.A., Kennedy, J., Blacque, O.E., Leroux, M.R., 2013. Striated rootlet and nonfilamentous forms of rootletin maintain ciliary function. Curr Biol 23, 2016-2022.

Muto, Y., Yoshioka, T., Kimura, M., Matsunami, M., Saya, H., Okano, Y., 2008. An evolutionarily conserved leucine-rich repeat protein CLERC is a centrosomal protein required for spindle pole integrity. Cell Cycle 7, 2738-2748.

Nokhbatolfoghahai, M., Downie, J.R., Ogilvy, V., 2006. Surface ciliation of anuran amphibian larvae: persistence to late stages in some species but not others. J Morphol 267, 1248-1256.

Ochi, T., Quarantotti, V., Lin, H., Jullien, J., Rosa, E.S.I., Boselli, F., Barnabas, D.D., Johnson, C.M., McLaughlin, S.H., Freund, S.M.V., Blackford, A.N., Kimata, Y., Goldstein, R.E., Jackson, S.P., Blundell, T.L., Dutcher, S.K., Gergely, F., van Breugel, M., 2020. CCDC61/VFL3 Is a Paralog of SAS6 and Promotes Ciliary Functions. Structure 28, 674-689 e611.

Park, T.J., Haigo, S.L., Wallingford, J.B., 2006. Ciliogenesis defects in embryos lacking inturned or fuzzy function are associated with failure of planar cell polarity and Hedgehog signaling. Nat Genet 38, 303-311.

Park, T.J., Mitchell, B.J., Abitua, P.B., Kintner, C., Wallingford, J.B., 2008. Dishevelled controls apical docking and planar polarization of basal bodies in ciliated epithelial cells. Nat Genet 40, 871-879.

Pizon, V., Gaudin, N., Poteau, M., Cifuentes-Diaz, C., Demdou, R., Heyer, V., Reina San Martin, B., Azimzadeh, J., 2020. hVFL3/CCDC61 is a component of mother centriole subdistal appendages required for centrosome cohesion and positioning. Biol Cell 112, 22-37.

Robert, J., Ohta, Y., 2009. Comparative and developmental study of the immune system in Xenopus. Dev Dyn 238, 1249-1270.

Sandoz, D., Chailley, B., Boisvieux-Ulrich, E., Lemullois, M., Laine, M.C., Bautista-Harris, G., 1988. Organization and functions of cytoskeleton in metazoan ciliated cells. Biol Cell 63, 183-193.

Satir, P., Heuser, T., Sale, W.S., 2014. A Structural Basis for How Motile Cilia Beat. Bioscience 64, 1073-1083.

Silflow, C.D., LaVoie, M., Tam, L.W., Tousey, S., Sanders, M., Wu, W., Borodovsky, M., Lefebvre, P.A., 2001. The Vfl1 Protein in Chlamydomonas localizes in a rotationally asymmetric pattern at the distal ends of the basal bodies. J Cell Biol 153, 63-74.

Spassky, N., Meunier, A., 2017. The development and functions of multiciliated epithelia. Nat Rev Mol Cell Biol 18, 423-436.

Tasca, A., Helmstadter, M., Brislinger, M.M., Haas, M., Mitchell, B., Walentek, P., 2020. Notch signaling induces either apoptosis or cell fate change in multiciliated cells during mucociliary tissue remodeling. Dev Cell.

Tateishi, K., Nishida, T., Inoue, K., Tsukita, S., 2017. Three-dimensional Organization of Layered Apical Cytoskeletal Networks Associated with Mouse Airway Tissue Development. Sci Rep 7, 43783. 
Turk, E., Wills, A.A., Kwon, T., Sedzinski, J., Wallingford, J.B., Stearns, T., 2015. ZetaTubulin Is a Member of a Conserved Tubulin Module and Is a Component of the Centriolar Basal Foot in Multiciliated Cells. Curr Biol 25, 2177-2183.

Walentek, P., Boutin, C., Kodjabachian, L., 2017. Planar cell polarity in ciliated epithelia, in: Houston, D. (Ed.), Cell Polarity in Development and Disease Elsevier.

Walentek, P., Quigley, I.K., Sun, D.I., Sajjan, U.K., Kintner, C., Harland, R.M., 2016. Ciliary transcription factors and miRNAs precisely regulate $\mathrm{Cp} 110$ levels required for ciliary adhesions and ciliogenesis. Elife 5.

Werner, M.E., Hwang, P., Huisman, F., Taborek, P., Yu, C.C., Mitchell, B.J., 2011. Actin and microtubules drive differential aspects of planar cell polarity in multiciliated cells. J Cell Biol 195, 19-26.

Woodruff, J.B., Wueseke, O., Hyman, A.A., 2014. Pericentriolar material structure and dynamics. Philos Trans R Soc Lond B Biol Sci 369.

Wright, R.L., Chojnacki, B., Jarvik, J.W., 1983. Abnormal basal-body number, location, and orientation in a striated fiber-defective mutant of Chlamydomonas reinhardtii. J Cell Biol 96, 1697-1707.

Yang, J., Gao, J., Adamian, M., Wen, X.H., Pawlyk, B., Zhang, L., Sanderson, M.J., Zuo, J., Makino, C.L., Li, T., 2005. The ciliary rootlet maintains long-term stability of sensory cilia. Mol Cell Biol 25, 4129-4137.

Yang, J., Li, T., 2005. The ciliary rootlet interacts with kinesin light chains and may provide a scaffold for kinesin-1 vesicular cargos. Exp Cell Res 309, 379-389.

Yasunaga, T., Hoff, S., Schell, C., Helmstadter, M., Kretz, O., Kuechlin, S., Yakulov, T.A., Engel, C., Muller, B., Bensch, R., Ronneberger, O., Huber, T.B., Lienkamp, S.S., Walz, G., 2015. The polarity protein Inturned links NPHP4 to Daam1 to control the subapical actin network in multiciliated cells. J Cell Biol 211, 963-973.

Zhang, S., Mitchell, B.J., 2015. Basal bodies in Xenopus. Cilia 5, 2.

\section{Acknowledgements}

Imaging in IBDM was performed on PiCSL-FBI core facility, supported by the French

National Research Agency through the program "Investments for the Future" (FranceBioImaging, ANR-10-INBS-04). The authors thank Florian Roguet for Xenopus care, Rémi Flores-Flores for ImageJ macro development, Brice Detailleur for 3D printing of embryo holders, Fabrice Richard, Nicolas Brouilly and Aicha Aouane from IBDM electron microscopy facility. This project was funded by grants from ANR (Oricen, 15-CE13-0003-02) and FRM (EQU201903007834). AN was supported by MESRI and FRM. 


\section{Authors contribution}

AN performed and analyzed most experiments. $\mathrm{CB}$, OR, EB, and LK analyzed experiments. OR generated and characterized Pcnt antibodies. EB wrote scripts to analyze BB spacing. VT performed and analyzed Pcnt immunostaining. AV and EL designed, and EL performed and analyzed cilia beating experiments. AN and CB drafted the original manuscript. LK edited the manuscript. LK designed and supervised the study and obtained funding. All authors commented on the manuscript.

\section{Competing financial interests}

The authors declare no competing financial interests.

\section{Figure legends}

Figure 1. Llrce1 and Ccdc61 associate with centrioles in multiciliated cells.

(A-C) MIPs (maximum intensity projections) of confocal acquisitions of MCCs stained with Lrrcc1 (green) and Centrin (red) antibodies. White dashed boxes indicate higher magnification views presented below and the dashed white line sets the position of the $\mathrm{Z}$ view. (D) MIPs of confocal acquisitions showing the localization of Lrrcc1 (green) compared to Centrin (red) and $\gamma$-Tubulin (white). White dotted boxes 1 and 2 indicate the BBs analysed in 3D with Clear Volume. (E-G) MIPs of confocal acquisitions from MCCs expressing Ccdc61-GFP (green) and Centrin-RFP (red) fusion proteins. (H) Clear volume 3D top and lateral views of a BB labelled with Ccdc61-GFP and Centrin-RFP. (I) Scheme of a mature BB in a Xenopus epidermal MCC recapitulating the localization of proteins analysed in this figure.

\section{Figure 2. Lrrec1 and Cedc61 are necessary for BB docking, spacing and orientation.}

(A) MIPs of confocal acquisitions of MCCs from St 31 control, Lrrcc1 or Ccdc61 morphant embryos stained with Centrin Ab, or expressing Centrin-RFP, which was co-injected with MOs. (B) Graph displaying the apico basal (A-B) distribution of centrioles within control, Lrrcc1 and Ccdc61 morphant 
and rescued MCCs. Each point represents a single cell. Horizontal lines represent the mean and SD. (C) MIPs of confocal acquisitions of apical BBs in MCCs from St 31 control, Lrrcc1 or Ccdc61 morphant embryos stained with Centrin Ab, or expressing Centrin-RFP, which was co-injected with MOs. The corresponding Delaunay triangulation outputs are presented on the bottom row. Each colour is associated to a range of triangle areas in $\operatorname{pixel}^{2}$. (D) Violin plots displaying the distribution of triangle areas in $\mu \mathrm{m}^{2}$ of control, morphant and rescued MCCs. The horizontal line indicates the median, dashed lines indicate the quartiles. (E) MIPs of confocal acquisitions of control and morphant MCCs stained with Centrin (red, centrioles) and $\gamma$-Tubulin (white, BF). Below each cell, its respective rose histogram representing the distribution of $\mathrm{BB}$ orientations. The black line running from the centre of the diagram to the outer edge is the mean angle and the arcs extending to either side represent the confidence limits of the mean fixed at $95 \%$ (when the mean angle is only theoretical but not significant, the line turns pink). (F) Circular Standard Deviations of control, morphant and rescued MCCs. Each point represents a single cell. Horizontal lines represent the mean and SD. (G) Graph displaying the percentage of nonpolarized MCCs (no significant mean angle of BBs within a cell can be calculated) following the Rayleigh statistical test. All confocal images are at the scale shown in (A).

\section{Figure 3. Ccdc61 is required for Pericentrin association to rootlet appendages.}

(A) Transversal TEM acquisitions of BBs from St 31 control and morphant MCCs. Both fan-shape (blue arrow) and long (red arrow) rootlets could be observed in all conditions, but rarely on the same section. (B) Quantification of BB docking on TEM acquisitions to corroborate morpholino efficiency. (C) Graph displaying the proportion of BBs with at least one (fan-shaped or long) or without rootlets quantified on TEM acquisitions. Please note that about $20 \%$ control BBs appear to lack rootlets due to the angle of the section. The same proportion was observed in Lrrcc1 and Ccdc61 morphant MCCs. (D) MIPs of confocal acquisitions of MCCs stained with Pcnt (green), Centrin (red) and $\gamma$-Tubulin (white). The white dashed box indicates the BB analysed in 3D in E. (E) Clear Volume 3D top and lateral views of a single BB. (F) Scheme of a mature BB recapitulating the localization of proteins analysed in this figure. (G) MIPs of confocal acquisitions of St 31 Lrrce1 and Ccdc61 morphant MCCs expressing 
Centrin-RFP (red) and stained with Pcnt antibody (green). White dashed boxes indicate high magnification views displayed on the right. (H) Quantification of Pcnt mean signal intensity. Each point represents an MCC. Horizontal lines represent the mean and SD.

Figure 4. Depletion of Lrrec1 and Cede61 impairs apical and subapical actin networks in MCCs. (A-C) MIPs of confocal acquisitions of St31 control and morphant MCCs stained for F-actin (white, Sir-actin), Centrin (red, Ab in control, Centrin-RFP co-injected with MOs to mark morphant MCCs). Dashed boxes indicate high magnification views in A'-C'. (A'-C') Single slices of confocal acquisitions showing the apical or subapical $(0.6 \mu \mathrm{m}$ below $)$ actin network in control and morphant cells. The dashed white line sets the position of the corresponding $\mathrm{Z}$ view (bottom row). (D-E) Graph displaying the quantification of mean F-actin signal intensity in control and morphant MCCs. Horizontal lines represent the mean and SD.

\section{Figure 5. Depletion of Lrrec1 and Cedc61 causes apical MT network disorganization in MCCs.}

(A-E) MIPs of confocal acquisitions of St 31 control and morphant MCCs after deciliation stained with $\alpha$-Tubulin (green, MTs) and Centrin Ab or expressing Centrin-RFP co-injected with MOs (red, BBs). White dashed boxes indicate the high magnification views in A'-E'. (A', C', E') Apical confocal slices in control (A'), Lrrce1 (C') and Ccde61 (E') morphant cells. The regular MT network that links BBs in control cells appear irregular in morphant conditions. (B', D') MIPs of confocal acquisitions showing intense $\alpha$-Tubulin signal around clustered BBs in Lrrcc1 and Ccde61 morphant MCCs. Dashed white lines set the position of the corresponding $\mathrm{Z}$ views shown on the bottom row.

\section{Figure 6. Depletion of Lrrce1 and Ccdc61 impairs apical intermediate filament network in MCCs.} (A-D) MIPs of confocal acquisitions of St 18 control MCC (A) and St 31 control (B) or morphant (CD) MCC stained for IFs (C-11 Ab, green) and BBs (Centrin Ab or Centrin-RFP co-injected with MOs, red). Dashed boxes indicate high magnification views in B'-D'. (B'-D') High magnification views of the zones boxed in B-D. The regular IF network that surrounds BBs in control cells appear much less 
dense in morphant conditions. White dashed lines set the position of the corresponding $Z$ views shown on the bottom row.

Figure 7. Lrrec1 and Cede61 depletion impairs ciliary beating, flow production and embryo resistance against pathogens.

(A) Quantification of cilia beating frequency (Hz) in control and morphant MCCs. Each dot represents the mean ciliary beat frequency computed over all visible cilia per individual MCC . Horizontal lines represent the mean and SD. (B) Still frames at 4 time-points taken from movie 3 showing the progression of the red dye along the flanks of control, Lrrcc1 and Ccdc61 morphant tadpoles. The black arrow represents the flow along the anterior-posterior axis (A-P). (C) Percentage of the embryo length reached by the dye front in 12 seconds. Each bar represents one recorded embryo. Cases marked with an asterisk are those shown in B. (D) Quantification of control and morphant tadpoles survival in presence or not of $A$. hydrophila bacteria.

\section{Video legends}

Video 1: Examples of Lrrec1 and Cedc61 morphant MCCs with partially preserved ciliary beating

Cilia beating was recorded at $250 \mathrm{fps}$, and the video is played at $50 \mathrm{fps}$ to better appreciate cilia movement.

Video 2: Examples of Lrrec1 and Cedc61 morphant MCCs without ciliary beating Cilia beating was recorded at $250 \mathrm{fps}$, and the video is played at $50 \mathrm{fps}$ to better appreciate cilia movement.

Video 3: Analysis of fluid flow in control, Lrrec1 and Ccde61 morphant embryos 
The video is played at real speed. Timing started when red microspheres were deposited onto the embryos.

\section{Supplementary figure legends}

Figure S1. Irrcc1 and ccdc61 transcript expression in Xenopus laevis embryos.

(A) In-situ hybridization for lrrccl and $c c d c 61$ at St 20. (B) 3D Clear Volume top and lateral views of two individual BBs stained with $\gamma$-Tubulin (white) and Centrin (red) antibodies. $\gamma$-Tubulin protein localizes to BF and rootlets in Xenopus epidermal MCCs.

\section{Figure S2. Lrrec1 and Ccde61 morpholino-mediated knock-down}

(A) Schematic representation of Xenopus lrrccl and $c c d c 61$ pre-mRNAs with introns and exons relative position and size. Red bars below exon1 show the position of $c c d c 61$ and lrrccl MO ATG and Spl. (B) MIPs of confocal acquisitions of St31 control or morphant MCCs stained for Centrin (centrioles, red), Lrrcc1 (green) and mRFP (tracer co-injected with MO, white). MO efficiency is validated by disappearance of the Ab staining. (C) MIPs of confocal acquisitions of St 31 control or morphant MCCs expressing Ccdc61-GFP (green) and stained for Centrin (red) and mRFP (white). MO efficiency is validated by extinction of Ccdc61-GFP.

\section{Figure S3. Lrrec1 and Cede61 knockdown impairs BB docking, spacing and orientation}

(A) MIPs of confocal acquisitions of MCCs from St 31 control, Lrrce1 Spl or Ccdc61 Spl morphant embryos stained with Centrin Ab, or Centrin-RFP co-injected with MOs (red). Dashed lines set the position of the corresponding $\mathrm{Z}$ views presented below. (B) Graph displaying the number of $\mathrm{Z}$ slices containing BBs in control, Lrrcc1 and Ccdc61 morphant and rescued embryos. Each point represents a single cell. Horizontal lines represent the mean and SD. (C) MIPs of confocal acquisitions of apical BBs in MCCs from St 31 control, Lrrcc1 or Cedc61 morphant embryos stained with Centrin Ab or CentrinRFP co-injected with MOs (red) and Delaunay triangulation outputs (bottom row). Each colour is associated to a range of triangle areas in pixel $^{2}$. (D) Violin plots displaying the distribution of triangle 
areas in $\mu \mathrm{m}^{2}$ of control, morphant and rescued MCCs. The horizontal line indicates the median, dashed lines indicate the quartiles. (E) MIPs of confocal acquisitions of control and morphant MCCs stained with Centrin (red, centrioles) and $\gamma$-Tubulin (white, BF). Below each cell, its respective rose histogram representing the distribution of $\mathrm{BB}$ orientations. The black line running from the centre of the diagram to the outer edge is the mean angle and the arcs extending to either side represent the confidence limits of the mean (when the mean angle is only theoretical but not significant, the line turns pink). (F) Graph displaying the percentage of non-polarized MCCs (no significant mean angle of BBs within a cell can be calculated), as revealed by the Rayleigh statistical test. (G) Circular Standard Deviations of control, morphant and rescued MCCs. Each point represents a single cell. Horizontal lines represent the mean and SD. All confocal images are at the scale shown in (A).

\section{Figure S4. Ciliogenesis is not affected by Lrrce1 and Ccdc61 depletion.}

MIPs of confocal acquisitions of St 31 control and morphant MCCs stained for centrioles (Centrin Ab, red), cilia (Acetylated- $\alpha$-tubulin Ab, green) and morpholino tracer (mRFP co-injected with MOs, white). White dashed lines indicate morphant MCCs.

Figure S5. Correct BB organization is rescued upon Lrrce1-GFP and Ccdc61-GFP mRNA coinjection in morphant MCCs.

(A-D) Embryos were sequentially injected first with MO + Centrin-RFP and then with Lrrcc1-GFP or MOresCcdc61-GFP mRNA (green). (A, C) MIPs of confocal acquisitions of St 31 MCCs expressing Centrin-RFP (BBs, tracer for MO injection, red), immunostained for BF ( $\gamma$-tubulin, white). The absence of specific signal in the green channel identifies those cells as morphant MCCs, consistent with severe BB disorganization. (B, D) MIPs of confocal acquisitions of MCCs expressing Centrin-RFP co-injected with MOs (red), Lrrcc1-GFP or MOresCcdc61-GFP (green) and immunostained for BF (white). In GFPpositive morphant MCCs, BB organization is largely rescued and comparable to control (see Fig. 2 and S3). 


\section{Figure S6. Pericentrin antibody validation.}

(A) Schematic representation of Xenopus laevis Pcnt protein. A portion of the protein between aa 33403643 was used as immunogen to generate rabbit polyclonal Ab. (B) COS1 cells were transfected or not (control) with vectors coding for the indicated proteins and immunoblotted with custom-made Pcnt rabbit $\mathrm{Ab}$, or $\mathrm{Ab}$ against GFP. The expected size of GFP-Pcnt is $62 \mathrm{kDa}$. (C) Clear Volume 3D top and lateral views of a single immunostained BB, showing the localization of Pcnt (green), relative to Centrin (red), and $\gamma$-Tubulin (white).

\section{Figure S7. Deciliation of Xenopus epidermal MCCs}

MIPs of confocal acquisitions of the ciliated epidermis before and after dibucaine treatment. Cilia and MTs are stained with $\alpha$-Tubulin Ab (green). The absence of cilia upon dibucaine treatment allows the observation of cytoplasmic MTs.

Figure S8. Phenotype validation of embryos incubated with $A$. hydrophila.

MIPs of confocal acquisitions of epidermis of St 31 embryos, from the same experimental pool as those used for our survival assay (Fig. 7), stained for cilia ( $\alpha$-Tubulin Ab, green), centrioles (Centrin Ab, white) and MO tracer (mRFP Ab, red). The visible BB disorganization confirms MO efficiency.

\section{Materials and methods}

\section{Ethics statement}

All experiments were performed following the Directive 2010/63/EU of the European parliament and of the council of 22 September 2010 on the protection of animals used for scientific purposes and approved by the "Direction départementale de la Protection des Populations, Pôle Alimentation, Santé Animale, Environnement, des Bouches du Rhône " (agreement number F1305521). 


\section{RNA probes and whole mount in situ hybridization}

cDNA fragments from Xenopus laevis lrrcc1.L (entrez gene 431936), and ccdc61.L (entrez gene 734655) were amplified from commercial cDNA (Horizon discovery) by PCR using the following primers:

lrrcc1 forward: 5'-GCGAACGGACACAGACAGTA-3';

lrrcc1 reverse: 5'-GAATTCCATGGTAGTCAGCTCCTGC-3';

ccdc61 forward: 5'-GCGGCCGCAAGTGGAGGATGCTGTGACC-3';

ccdc61 reverse: 5'-GAATTCACGGATGAACTGCGTCTCTG-3'.

PCR products were cloned in pBlueScript KS+ vector and digoxigenin-labelled probes were generated from linearized plasmids using RNA-labeling mix (Roche). Whole-mount chromogenic in situ hybridization was performed as described previously (Marchal 2009) using 40ng of Digoxygenin-labelled probe. Pictures were taken with the stereomicroscope Leica MZ125 coupled to NIKON digital Sight DS-Fil camera.

\section{Plasmids, RNAs, and Morpholinos}

To generate plasmids for RNA synthesis and micro-injection, the ORFs of $l r r c c 1$ and $c c d c 61$ were amplified by PCR using the following primers:

lrrccl-forward: 5' TCTTTTTGCAGGATCACAATGGCAGGCACGGACCCACGAA 3'; lrrcc1-reverse: 5' CTTTACTCATTCTAGAAAATTCTTTTTGGATGTCACTTAG 3'; ccdc61-forward: 5' TCTTTTTGCAGGATCACAATGGAGGATACAGAGTTTGCT 3'; ccdc61-reverse: 5' CTTTACTCATTCTAGACTGCATCAGTAAGTACCCGCTGGCT 3'. PCR products were subcloned in frame with GFP sequence in 3' into pCS2+-GFP vector using In-Fusion ${ }^{\circledR}$ HD Cloning Kit (Takara Bio USA, Inc.). For rescue experiments, silent mutations were introduced by PCR in the original Ccdc61 sequence (3' GAG-GAT-ACA-GAG-TTT- 
GCT-GAA-G 5') to generate a Ccdc61-GFP construct (named MOresCcdc61-GFP) with a sequence resistant to MO ATG (3' GAA-GAC-ACG-GAA-TTC-GCT-GAA 5'). pCS2+-mRFP was used to generate an injection reporter.

The cDNA fragment coding for amino-acids 3340 to 3643 of the full-length Xenopus laevis Pcnt was amplified by PCR from a partial cDNA clone (IMAGE 5156155, Source Bioscience). PCR products were cloned by Gateway ${ }^{\circledR}$ recombination into pGEX6P3 (GE Healthcare Life Sciences) and pEGFP-C1 (Clontech) to produce GST fusion proteins and express GFP-tagged proteins, respectively. The Centrin-RFP plasmid was a kind gift from JB Wallingford.

Capped mRNAs were synthetized from linearized vectors using the SP6 mMESSAGE mMACHINE® Kit (Ambion Life Technologies) and purified with the MEGAclear ${ }^{\mathrm{TM}}$ Kit (Ambion Life Technologies).

Two independent morpholino antisense oligonucleotides were designed against lrrccl and ccdc61 (GeneTools, LLC). lrrcc1-ATG-MO: 5' GTGCCTGCCATTCTCCCGCAACAAA 3'; lrrccl-Spl-MO: 5' ACTGAAGCCATGCTGCTTACCTGGA 3'; ccdc61-ATG-MO: 5' CTTCAGCAAACTCTGTATCCTCCAT 3'; $\quad$ ccdc61-Spl-MO: $5^{\prime}$ TGTCTCCCACTTCTACTCACATTGA 3'.

\section{$\underline{\text { Xenopus embryo injections }}$}

Eggs obtained from NASCO females were fertilized in vitro, dejellied and cultured as described previously (Marchal et al., 2009). 20-30 ng of MO was injected (alone or with 200-500pg of mRFP tracer) in one or two animal-ventral blastomere (presumptive epidermis) at different stages depending on the experiment (Table 2). Working amounts of MOs were first calibrated to optimize embryo survival. Validation of MOs efficacy in depleting the target protein was confirmed based on Lrrcc1 antibody and Ccdc61-GFP signal disappearance. For rescue experiments, a sequential injection strategy was adopted to obtain mosaic embryos containing 
differentially marked morphant and rescued MCCs. At 4-cell stage, lrrccl and ccdc61-ATGMOs + Centrin-RFP or lrrccl and $c c d c 61-S p l-M O s+$ Centrin-RFP was injected At 16-cell stage, the same embryos were injected with Lrrcc1-GFP or MOresCcdc61-GFP mRNAs.

After injection, embryos were incubated at different temperatures between $13^{\circ}, 18^{\circ}$ and $23^{\circ} \mathrm{C}$ in MBS 0.1x until they reached the desired developmental stage. Whole-embryos were fixed in different conditions (Table 3) and stored in $100 \%$ methanol at $-20^{\circ} \mathrm{C}$ in preparation for immunofluorescent staining.

\section{$\underline{\text { Embryo deciliation }}$}

Stage 31 embryos were incubated for $2 \mathrm{~h}$ in a $35-\mathrm{mm}$ Petri dish containing $2 \mathrm{ml}$ of dibucaine

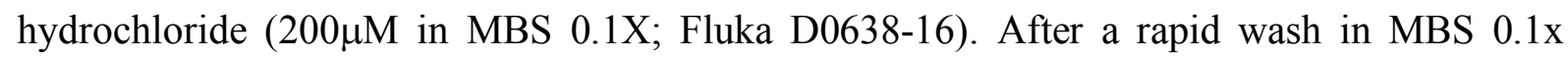
embryos were immediately fixed by incubation in $\mathrm{MetOH} 100 \%$ at $-20^{\circ} \mathrm{C}$ for two days before being further processed for immunofluorescent staining.

\section{Immunostaining}

After a sequential re-hydration in solutions with decreasing methanol concentrations, embryos were incubated in blocking solution (3\% Bovine Serum Albumin (BSA) in PBS 1x) for 1h at room temperature (RT) and subsequently in primary antibodies diluted in 3\% BSA overnight at $4^{\circ} \mathrm{C}$ (Table 2). After washing in PBS 1x embryos were incubated with fluorescently labelled secondary antibodies diluted in 3\% BSA for $1 \mathrm{~h}$ at RT (Table 4). After washing, embryos were mounted in Mowiol (Sigma-Aldrich) between slide and coverslip.

\section{Confocal Microscopy}

Confocal pictures were acquired using ZEISS LSM 780 right standing AxioImager Z2 and ZEISS LSM 880 reverse standing AxioObserver 7 equipped with 20x and 63x oil objectives. 
Two- or three-colors confocal z-series images (z-slices interval between $0.3-0.8 \mathrm{~nm}$ ) were acquired using sequential laser excitation. When necessary, images were converted into single plane maximum intensity projection (MIP) and edited using Image J 2.0.0 software.

\section{Image analysis}

F-actin and Pcnt signals were quantified by measuring the mean pixel intensity per MCC with ImageJ.

BB docking was quantified manually by counting the number of z-slices containing Centrin- or Centrin-RFP-positive centrioles along the apical-basal axis for each cell. These values were transformed in $\mu \mathrm{m}$ based on the interval size between slices set during confocal acquisition.

BB spacing analysis was performed from Centrin or Centrin-RFP immunostaining images using custom made Matlab scripts. After manual segmentation of individual cells, the script (i) automatically detects, and segment individual BBs within each cell. Only apically docked and isolated BBs are considered, omitting those located below the apical cell membrane or in dense clusters; (ii) determine the centroid of each BB; (iii) use XY coordinates of all the centroids to build triangles between the nearest neighbors with the Delaunay Triangulation Matlab script. Once the triangulation is obtained, areas of the triangles are measured in pixel $^{2}$ and transformed in $\mu \mathrm{m}^{2}$ based on the pixel size of each acquisition.

BB orientation was analysed using a home-made ImageJ script (designed by R. Flores-Flores). The script (i) automatically detects individual MCCs in the field of view; (ii) automatically detects individual BBs in each MCC; (iii) traces a vector from Centrin to $\gamma$-Tubulin spots (a threshold is applied for $\gamma$-Tubulin channel in order to detect only the BF associated spot, and not the rootlet spots) for each BB and calculate its angle with the vertical axis. The output of the script is a list of angles that were plotted using the Oriana software (Version 4.02, Kovach Computing Services) to obtain a graphical representation of their distribution in $25,7^{\circ}$ bins, with 
$95 \%$ of confidence intervals. Finally, circular statistical analysis were performed (CSD, Rayleigh's Uniformity Test).

\section{Transmission electron microscopy}

Embryos were processed for electron microscopy as previously described (Revinski 2018) and cut transversely at midbody level (80 nm/slice) with a Leica Ultracut UC7 (Leica, Germany). Images were acquired using a Tecnai G2 (Thermofisher, USA) microscope equipped with a Veleta camera (Olympus, Japan).

\section{Cell culture and Western Blot}

Cos-1 cells were grown in DMEM supplemented with $10 \%$ heat inactivated FCS and transfected with Fugene HD (Roche Applied Science) according to manufacturer's protocol. Transfected or control cells were washed in PBS and lysed in $50 \mathrm{mM}$ Tris $\mathrm{HCl}$ pH 7.5, $150 \mathrm{mM}$ $\mathrm{NaCl}, 1 \mathrm{mM}$ EDTA, containing $1 \% \mathrm{NP}-40$ and $0.25 \%$ sodium deoxycholate (modified RIPA) plus a Complete Protease Inhibitor Cocktail (Roche Applied Science) on ice. Cell extracts separated on polyacrylamide gels were transferred onto Optitran membrane (Whatman), followed by incubation with rabbit anti-GFP (1:5000, Abcam, ab290), or homemade rabbit antiPcnt antibody $(1 \mu \mathrm{g} / \mathrm{ml})$ produced by immunization with recombinant portion of Xenopus laevis Pcnt (XP_018091513.1, residues 3340-3643), and horseradish peroxidase-conjugated secondary antibody (Jackson Immunoresearch Laboratories, 711-035-152). Signal obtained from enhanced chemiluminescence (Western Lightning ECL Pro, Perkin Elmer) was detected with MyECL Imager (ThermoFisher Scientific). 


\section{Flow and cilia beating measurements}

Stage 31 living embryos were placed in anaesthetic solution media (MS222 0.02\% in MBS $0.1 x$ ) in a home-made 3D-printed Petri Dish specially designed to maintain them with the dorsal side on the top. The setup was placed under compact Stereo Microscope ZEISS Stemi 305 coupled to ZEISS Axiocam 105 Color microscope camera. Timelapse recording was done after release of $1 \mu 1$ of visible dyed microspheres (Bangs Laboratories: DSCR006, Mean diameter $5.19 \mu \mathrm{m}$, diluted $1 / 2$ in MBS $0,1 \mathrm{x})$ at the anterior front of the embryo.

Cilia beating frequency was analysed on the same pool of living embryos. Embryos were placed between a glass slide and a coverslip with a drop of anaesthesic medium (MBS 0.1x - MS222 $0.02 x$ ) surrounded by grease (high vacuum grease DOW CORNING, Sigma-Aldrich Z2735541EA) to stick the coverslip. Movies with a duration of 3 seconds (250 fps) were recorded at the ventral border of the embryos with a Nikon eclipse Ti-E microscope and a $63 x$ long working distance air objective. Computation of cilia beat frequency (CBF) was done using an in-house routine developed in python (Khelloufi et al. 2018). The resulting frequencies (Hz) shown in the quantification represent the mean CBF computed over all visible cilia per individual MCC. Movies are played at 50fps to observe better the beating strokes and defaults. After flow and CBF recording, MO efficiency was assessed in those embryos by immunostaining for Centrin, Acetylated- $\alpha$-tubulin and mRFP (injection tracer).

\section{Embryo survival to Aeromonas hydrophila}

Aeromonas hydrophila (Chester Stanier, ATCC®7966 kindly provided by E. Dubaissi) colonies were grown for $48 \mathrm{~h}$ at $37^{\circ} \mathrm{C}$ on LB Agar $+30 \mu \mathrm{g} / \mathrm{mL}$ Kanamycin. Next, individual colonies were cultured in $10 \mathrm{~mL} \mathrm{LB}+30 \mu \mathrm{g} / \mathrm{mL}$ Kanamycin overnight at $37^{\circ} \mathrm{C}$. The $\mathrm{OD}_{600}$ (OD600 DiluPhotometer version1.4, Implen) was measured and the culture was either diluted in $\mathrm{LB}$ or grown further to reach an $\mathrm{OD}_{600}$ of 0.2 . Cultures were centrifuged $10 \mathrm{~min}$ at $3500 \mathrm{xg}$ and the bacteria pellet was resuspended in the same volume of MBS 0.1x. Controls (Non- 
injected or mRFP-injected) and morphant (Lrrcc1-MO+mRFP or Ccdc61-MO+mRFP) embryos were incubated from St 31 during $72 \mathrm{~h}$ at $13^{\circ} \mathrm{C}$ in $3 \mathrm{~mL}$ of bacteria containing medium. Survival was assessed by recording active response to touch at $2 \mathrm{~h}, 4 \mathrm{~h}, 24 \mathrm{~h}, 48 \mathrm{~h}$ and $72 \mathrm{~h}$.

At the end of the experiment, MO efficiency was assessed by immunostaining for Centrin, Acetylated- $\alpha$-tubulin and mRFP tracer.

\section{$\underline{\text { Statistics }}$}

For all experiments, statistical analysis of significance was done using GraphPad Prism (version 8.2.0 for Windows, GraphPad Software, San Diego, California USA). First, the normality of data (Gaussian distribution) was tested using D'agostino \& Pearson test (if $n>50$ ) or ShapiroWilk test (if $\mathrm{n}<50$ ). When the data followed a normal distribution, we then compared them using parametric Student t-tests (between 2 groups) or one One-way ANOVA (between $>2$ groups). When data did not follow a normal distribution, we compared them using MannWhitney (2 groups) or Kruskal-Wallis ( $>2$ groups) non-parametric tests. $p=0.0001-0.001$ or $<0.0001$ was considered extremely significant $(* * *), p=0.001-0.01$ was considered very significant $(* *) ; p=0.01-0.05$ was considered significant $(*)$ and $p=\geq 0.05$ was considered not significant (ns). Number of embryos and experiments performed for all analysis are listed in table 5.

\section{Tables}

\begin{tabular}{|c|c|c|c|c|}
\hline Gene name (bait) & Gene Name (prey) & Enrichment & $\begin{array}{c}\text { Linked to } \\
\text { centriole/cilia/MTs }\end{array}$ & $\begin{array}{c}\text { Found } \\
\text { in } \\
\text { Xenopus } \\
\text { MCCs }\end{array}$ \\
\hline LRRCC1 & LRRCC1 & 10,59121 & YES & YES \\
\hline LRRCC1 & CCDC77 & 9,79952 & PROBABLE & YES \\
\hline LRRCC1 & AZI1/CEP131 & 9,645587 & YES & $?$ \\
\hline LRRCC1 & PCM1 & 8,385486 & YES & YES \\
\hline LRRCC1 & CEP72 & 8,169336 & YES & YES \\
\hline LRRCC1 & MIB1 & 6,578583 & YES & NO \\
\hline
\end{tabular}




\begin{tabular}{|c|c|c|c|c|}
\hline LRRCC1 & FOPNL/FOR20/CEP20 & 6,448221 & YES & YES \\
\hline LRRCC1 & BBS4 & 6,426498 & YES & YES \\
\hline LRRCC1 & OFD1 & 6,392701 & YES & YES \\
\hline LRRCC1 & WDR90 & 6,318789 & YES & YES \\
\hline LRRCC1 & KIAA0753/OFIP & 6,241836 & YES & YES \\
\hline LRRCC1 & CSPP1 & 6,139616 & YES & YES \\
\hline LRRCC1 & KIAA1731/CEP295 & 5,889853 & YES & YES \\
\hline LRRCC1 & PIBF1 & 5,812087 & YES & YES \\
\hline LRRCC1 & CCDC18 & 5,503085 & PROBABLE & YES \\
\hline LRRCC1 & WDR67 & 4,986441 & YES & $?$ \\
\hline LRRCC1 & CCDC61 & 4,976773 & YES & YES \\
\hline LRRCC1 & FGFR1OP/CEP43 & 4,853146 & YES & YES \\
\hline LRRCC1 & SSX2IP & 4,807299 & YES & yes \\
\hline LRRCC1 & CCDC14 & 4,215897 & YES & YES \\
\hline LRRCC1 & KIAA1328 & 3,94653 & YES & NO \\
\hline LRRCC1 & CEP350 & 3,833714 & YES & YES \\
\hline LRRCC1 & PHLDB2 & 3,801527 & YES & NO \\
\hline LRRCC1 & PRKAR2A & 3,785393 & YES & NO \\
\hline LRRCC1 & TTLL5 & 3,725921 & YES & NO \\
\hline LRRCC1 & N4BP3 & 3,684075 & NO & $?$ \\
\hline LRRCC1 & CEP170 & 2,879607 & YES & YES \\
\hline LRRCC1 & $\mathrm{ESCO} 2$ & 2,533443 & NO & $?$ \\
\hline LRRCC1 & SPICE1 & 2,417896 & YES & YES \\
\hline LRRCC1 & KIFC3 & 2,255614 & YES & NO \\
\hline FGFR1OP & CCDC61 & 7,169243 & YES & YES \\
\hline CEP120 & CCDC61 & 7,155219 & YES & YES \\
\hline OFD1 & CCDC61 & 5,799062 & YES & YES \\
\hline CEP72 & CCDC61 & 5,62468 & YES & YES \\
\hline MIB1 & CCDC61 & 5,100073 & YES & NO \\
\hline LRRCC1 & CCDC61 & 4,976773 & YES & YES \\
\hline CEP135 & CCDC61 & 4,941259 & YES & YES \\
\hline SDCCAG8 & CCDC61 & 4,656678 & YES & YES \\
\hline CEP152 & CCDC61 & 4,148394 & YES & YES \\
\hline KIF7 & CCDC61 & 3,749279 & YES & NO \\
\hline TPX2 & CCDC61 & 3,734188 & YES & NO \\
\hline NEDD1 & CCDC61 & 3,682512 & YES & YES \\
\hline CEP55 & CCDC61 & 3,550385 & YES & NO \\
\hline CCDC14 & CCDC61 & 3,546823 & YES & YES \\
\hline SLAIN2 & CCDC61 & 3,535149 & YES & NO \\
\hline PPP2CB & CCDC61 & 3,320352 & NO & NO \\
\hline PCM1 & CCDC61 & 3,265138 & YES & YES \\
\hline AZI1/CEP131 & CCDC61 & 2,932961 & YES & $?$ \\
\hline CSNK1E & CCDC61 & 2,845102 & NO & NO \\
\hline ANKRD26 & CCDC61 & 2,78639 & YES & YES \\
\hline
\end{tabular}




\begin{tabular}{|l|l|l|l|c|}
\hline PRKAR2A & CCDC61 & 2,684373 & YES & NO \\
\hline PPP2R5D & CCDC61 & 2,613387 & NO & $?$ \\
\hline ZBTB33 & CCDC61 & 2,575864 & NO & NO \\
\hline RAD54L & CCDC61 & 1,843647 & NO & NO \\
\hline SRPR & CCDC61 & 1,821545 & NO & NO \\
\hline
\end{tabular}

Table 1: Lrrec1 and Cedc61 interactors

This table has been annotated from the Human Interactome Purification and Mass

Spectrometry data of Hein et al., (2015). In green are shown common interactors between

Lrrcc1 and Ccdc61. Enrichment values represent the average enrichment of the protein in multiples of standard deviations compared to control samples. Published evidence was screened to identify the existence or not of links with centrioles, cilia or MTs. Transcript expression in Xenopus tropicalis MCCs is displayed, as revealed by scRNA-sequencing (Briggs et al., 2018). Question marks correspond to transcripts that were totally absent from the available dataset.

\begin{tabular}{|c|c|c|}
\hline mRNA & $\begin{array}{c}\text { Quantity injected per } \\
\text { blastomere (pg) }\end{array}$ & Stage of injection \\
\hline lrrccl-GFP & 300 & $8 / 16$ cells \\
\hline$c c d c 61-G F P$ & 300 & $4 / 8$ cells \\
\hline$m R F P$ & 200 & $4 / 8$ cells \\
\hline$c e n t r i n-R F P$ & 50 & $4 / 8$ cells \\
\hline$G F P-g p i$ & 500 & \\
\hline
\end{tabular}

Table 2 : mRNA injection conditions

\begin{tabular}{|c|c|c|c|}
\hline Fixation & Target/Species/Isotype & Reference & Dilution \\
\hline MetOH $100 \% 4^{\circ} \mathrm{C}$ & $\gamma$-Tubulin mouse IgG1 & Abcam Ab11316 & $1 / 800$ \\
\cline { 2 - 4 } OverWeekend or & Acetylated-tubulin mouse & Sigma-Aldrich T7451 & $1 / 1000$ \\
PFA 4\% 0,1\% & IgG2b & & \\
\cline { 2 - 4 } Triton 30min RT & GFP chicken & AvesLab GFP-1020 & $1 / 500$ \\
\cline { 2 - 4 } & RFP rat IgG2a & Chromotek 5F8 & $1 / 500$ \\
\cline { 2 - 4 } & & &
\end{tabular}




\begin{tabular}{|c|c|c|c|}
\hline & Centrin mouse IgG2a & Sigma-Aldrich 04-1624 & $1 / 1000$ \\
\hline & $\begin{array}{l}\text { Cytokeratin (Pan-reactive) } \\
\text { mouse monoclonal IgG1 }\end{array}$ & Sigma-Aldrich SAB4700666 & $1 / 500$ \\
\hline & Z01 mouse IgG1 & Thermo Scientifique 33-9100 & $1 / 200$ \\
\hline & $\alpha$-Tubulin mouse IgG1 & Sigma-Aldrich T9026 & $1 / 500$ \\
\hline Memfa 1h RT & Sir-Actin & Spirochrome SC001 & $1 / 1000$ \\
\hline $\begin{array}{l}\text { Memfa } 2 \mathrm{~h} \mathrm{RT} \text { or } \\
\text { OverNight } 4 \mathrm{C}^{\circ} \mathrm{C}\end{array}$ & Anti-DIG-PA conjugated IgG & Roche 11266026 & $1 / 500$ \\
\hline $\begin{array}{c}\text { PFA 4\% Triton } 0,1 \mathrm{x} \\
\text { 30min RT }\end{array}$ & Pericentrin rabbit & Home made, this study & $1 / 5000$ \\
\hline $\begin{array}{c}\text { MetOH } 100 \% 4^{\circ} \mathrm{C} \\
\text { OverWeekend }\end{array}$ & Lrrcc1 rabbit polyclonal & Sigma-Aldrich HPA012893 & $1 / 1000$ \\
\hline
\end{tabular}

Table 3: Primary antibodies and markers

\begin{tabular}{|l|l|l|}
\hline \multicolumn{1}{|c|}{ Antibody } & \multicolumn{1}{|c|}{ Reference } & Dilution \\
\hline Alexa anti mouse IgG2a 488 & Invitrogen Thermo Fisher A21131 & $1 / 800$ \\
\hline Alexa anti mouse IgG2a 568 & Invitrogen Thermo Fisher A21134 & $1 / 800$ \\
\hline Alexa anti mouse IgG2a 647 & Invitrogen Thermo Fisher A21241 & $1 / 800$ \\
\hline Alexa anti mouse IgG1 488 & Invitrogen Thermo Fisher A21121 & $1 / 800$ \\
\hline Alexa anti mouse IgG1 568 & Invitrogen Thermo Fisher A21124 & $1 / 800$ \\
\hline Alexa anti mouse IgG1 647 & Invitrogen Thermo Fisher A21240 & $1 / 800$ \\
\hline Alexa anti mouse IgG2b 488 & Invitrogen Thermo Fisher A21141 & $1 / 800$ \\
\hline Alexa anti mouse IgG2b 568 & Invitrogen Thermo Fisher A21144 & $1 / 800$ \\
\hline Alexa anti mouse IgG2b 647 & Invitrogen Thermo Fisher A21242 & $1 / 800$ \\
\hline Anti rat Cy3 IgG & Jackson ImmunoResearch 712165153 & $1 / 500$ \\
\hline Alexa fluor Anti rat 488 IgG & Life Technologies A21208 & $1 / 500$ \\
\hline Alexa fluor Anti rat 647 IgG & Jackson ImmunoResearch 712605153 & $1 / 500$ \\
\hline Alexa fluor Anti rabbit 488 IgG & Life Technologies A21206 & $1 / 500$ \\
\hline Alexa fluor Anti rabbit 568 IgG & Life Technologies A10042 & $1 / 500$ \\
\hline Alexa fluor Anti rabbit 647 IgG & Life Technologies A31573 & $1 / 500$ \\
\hline Alexa fluor Anti chicken 488 IgY & Jackson ImmunoResearch 703545155 & $1 / 500$ \\
\hline
\end{tabular}

Table 4: Secondary antibodies 


\begin{tabular}{|c|c|c|c|c|}
\hline Experiment & Condition & $\begin{array}{c}\text { Number of MCCs } \\
\text { (or BBs for TEM) } \\
\text { analyzed }\end{array}$ & $\begin{array}{c}\text { Number of } \\
\text { embryos } \\
\text { analyzed }\end{array}$ & $\begin{array}{l}\text { Number of } \\
\text { experimental } \\
\text { repeats }\end{array}$ \\
\hline \multirow{9}{*}{ BB orientation } & Control & 125 & \multirow{9}{*}{$4-9$} & \multirow{9}{*}{$2-3$} \\
\hline & Lrrcc1 ATG-MO & 24 & & \\
\hline & Lrrce1 Spl-MO & 28 & & \\
\hline & Ccdc61 ATG-MO & 33 & & \\
\hline & Ccde61 Spl-MO & 27 & & \\
\hline & Lrrcc1 ATG-MO +mRNA & 40 & & \\
\hline & Lrrcc1 Spl-MO +mRNA & 45 & & \\
\hline & Ccdc61 ATG-MO +mRNA & 39 & & \\
\hline & Ccdc61 Spl-MO +mRNA & 20 & & \\
\hline \multirow{5}{*}{$\begin{array}{c}\text { MCC } \\
\text { polarization } \\
\text { Rayleigh Test }\end{array}$} & Control & 591 & \multirow{5}{*}{ 4-9 } & \multirow{5}{*}{3} \\
\hline & Lrrcc1 ATG-MO & 181 & & \\
\hline & Lrrcc1 Spl-MO & 33 & & \\
\hline & Ccdc61 ATG-MO & 158 & & \\
\hline & Ccdc61 Spl-MO & 159 & & \\
\hline \multirow{9}{*}{ BB docking } & Control & 28 & \multirow{9}{*}{$3-9$} & \multirow{9}{*}{$2-3$} \\
\hline & Lrrcc1 ATG-MO & 36 & & \\
\hline & Lrrcc1 Spl-MO & 33 & & \\
\hline & Ccdc61 ATG-MO & 34 & & \\
\hline & Ccde61 Spl-MO & 28 & & \\
\hline & Lrrcc1 ATG-MO +mRNA & 41 & & \\
\hline & Lrrcc1 Spl-MO +mRNA & 35 & & \\
\hline & Ccdc61 ATG-MO +mRNA & 35 & & \\
\hline & Ccdc61 Spl-MO +mRNA & 29 & & \\
\hline \multirow{6}{*}{ BB spacing } & Control & 19 & \multirow[b]{6}{*}{2} & \multirow{6}{*}{$2-3$} \\
\hline & Lrrcc1 ATG-MO & 20 & & \\
\hline & Lrrcc1 Spl-MO & 28 & & \\
\hline & Ccdc61 ATG-MO & 27 & & \\
\hline & Ccdc61 Spl-MO & 11 & & \\
\hline & Lrrcc1 ATG-MO +mRNA & 43 & & \\
\hline
\end{tabular}




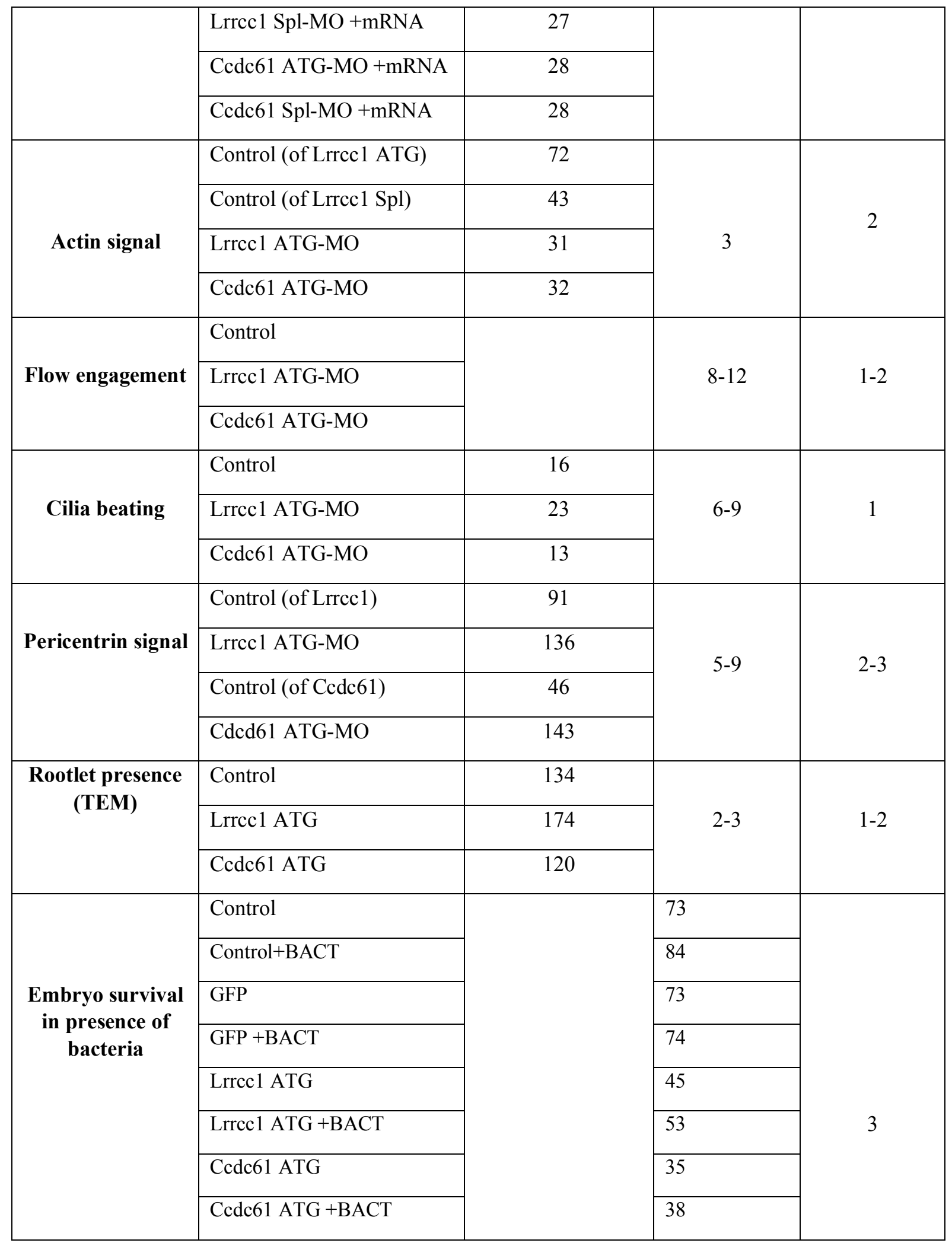

Table 5 : Experimental replicates and sample sizes 


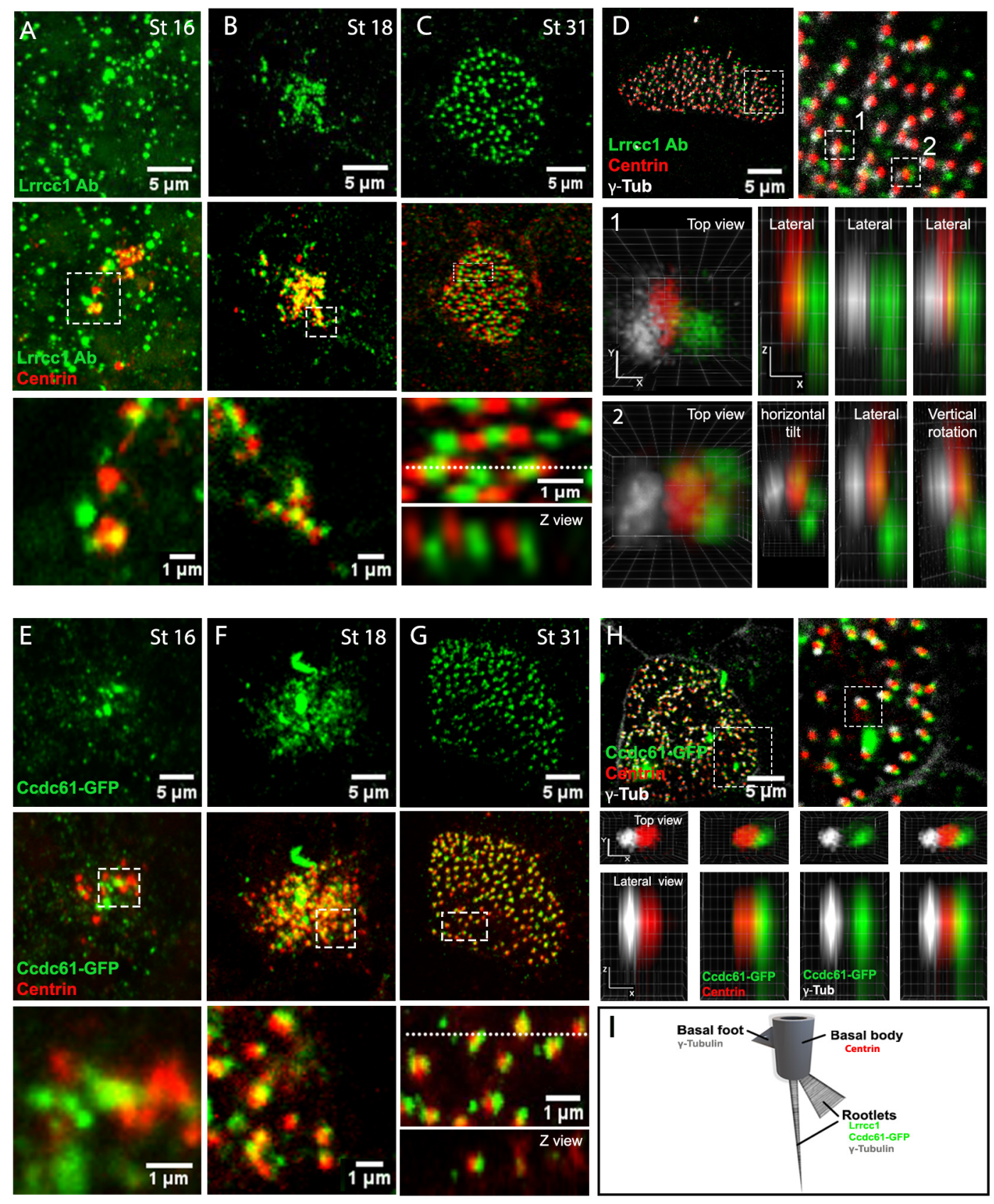




\section{A}

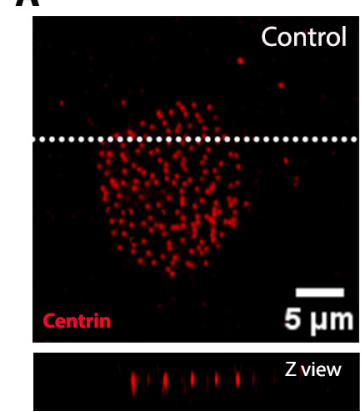

\section{C}

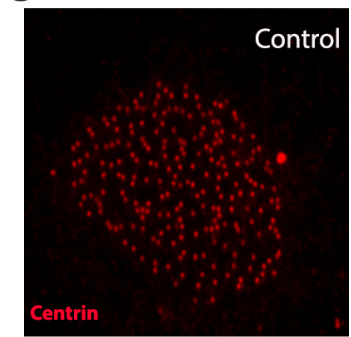

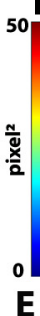
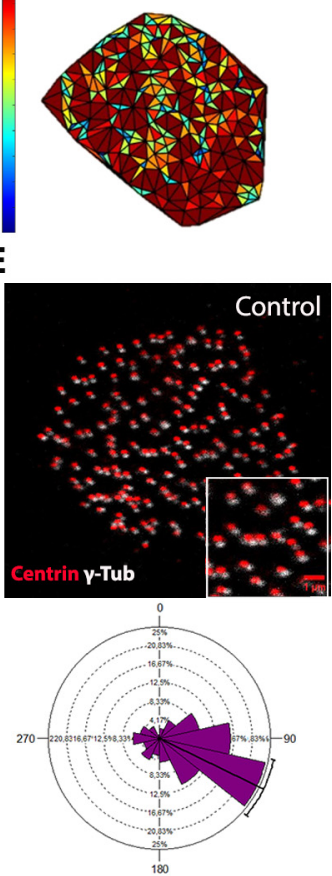

Basal Body DOCKING

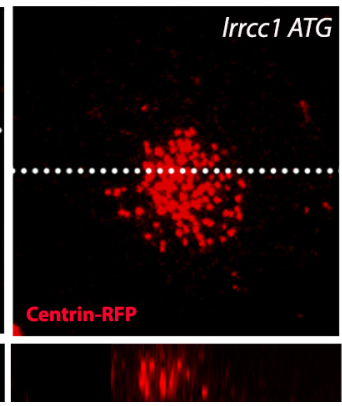

Basal Body SPACING
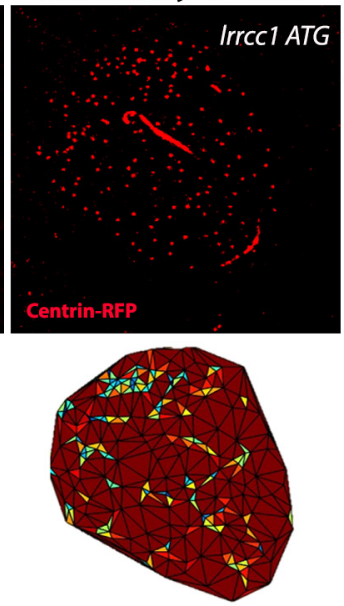

Basal Body POLARITY
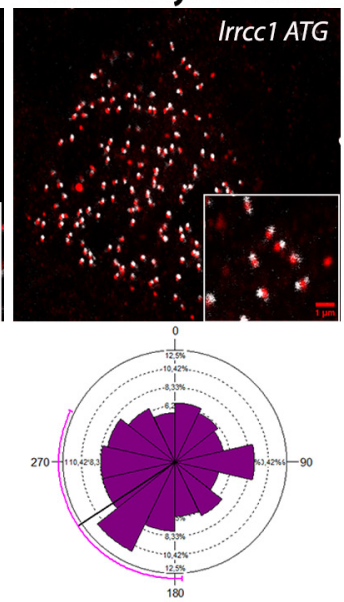

ccdc61 ATG
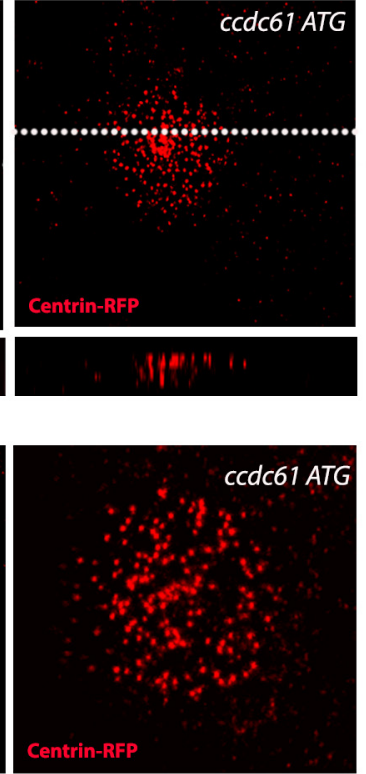

D
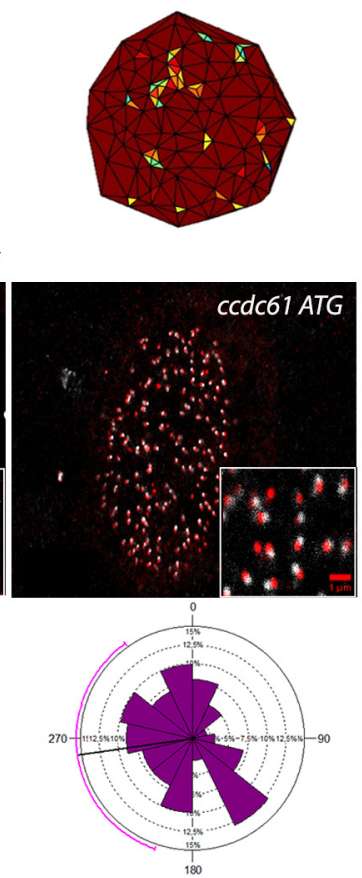

$F$
Basal body distribution along $A-B$ axis

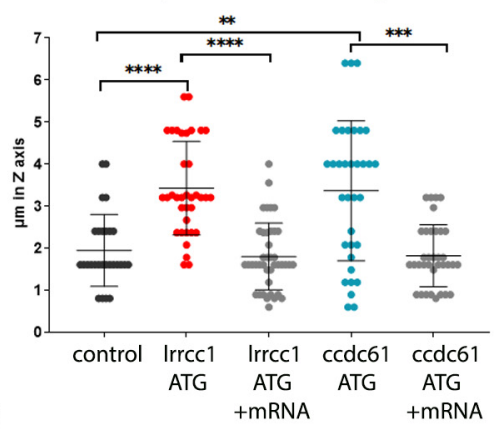

Basal body spacing
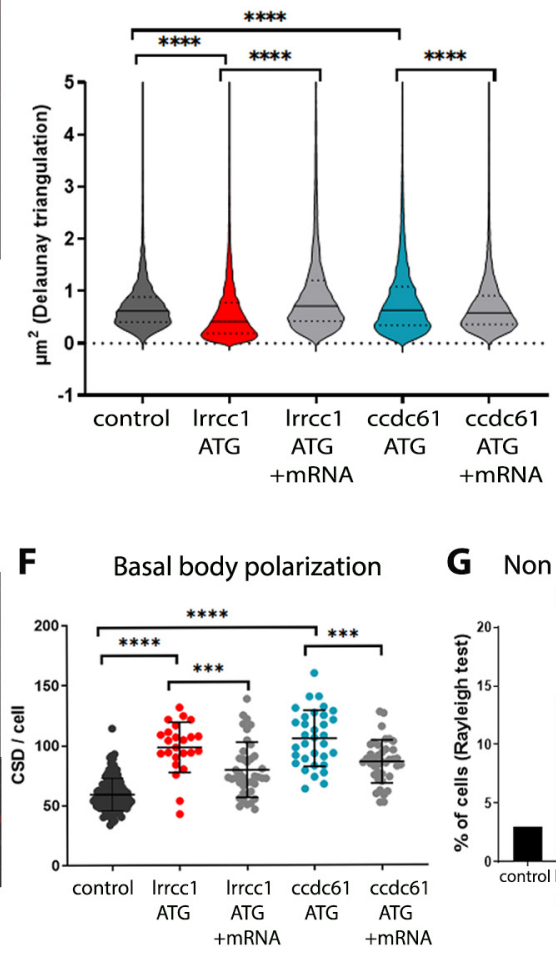

G Non polarized MCCs

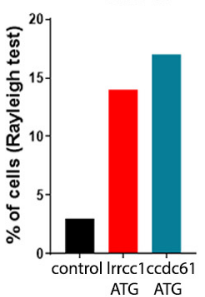



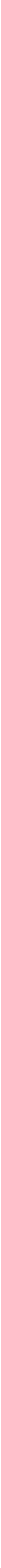

H PCNT signal intensity
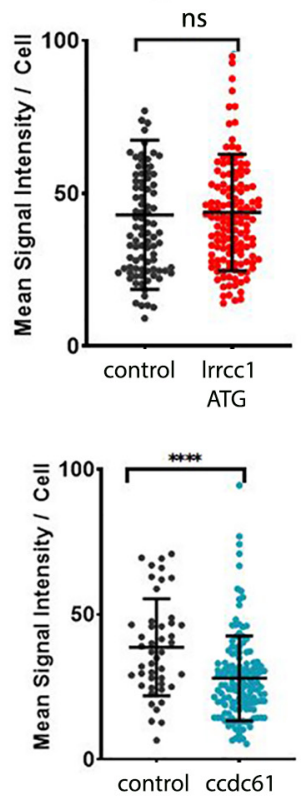

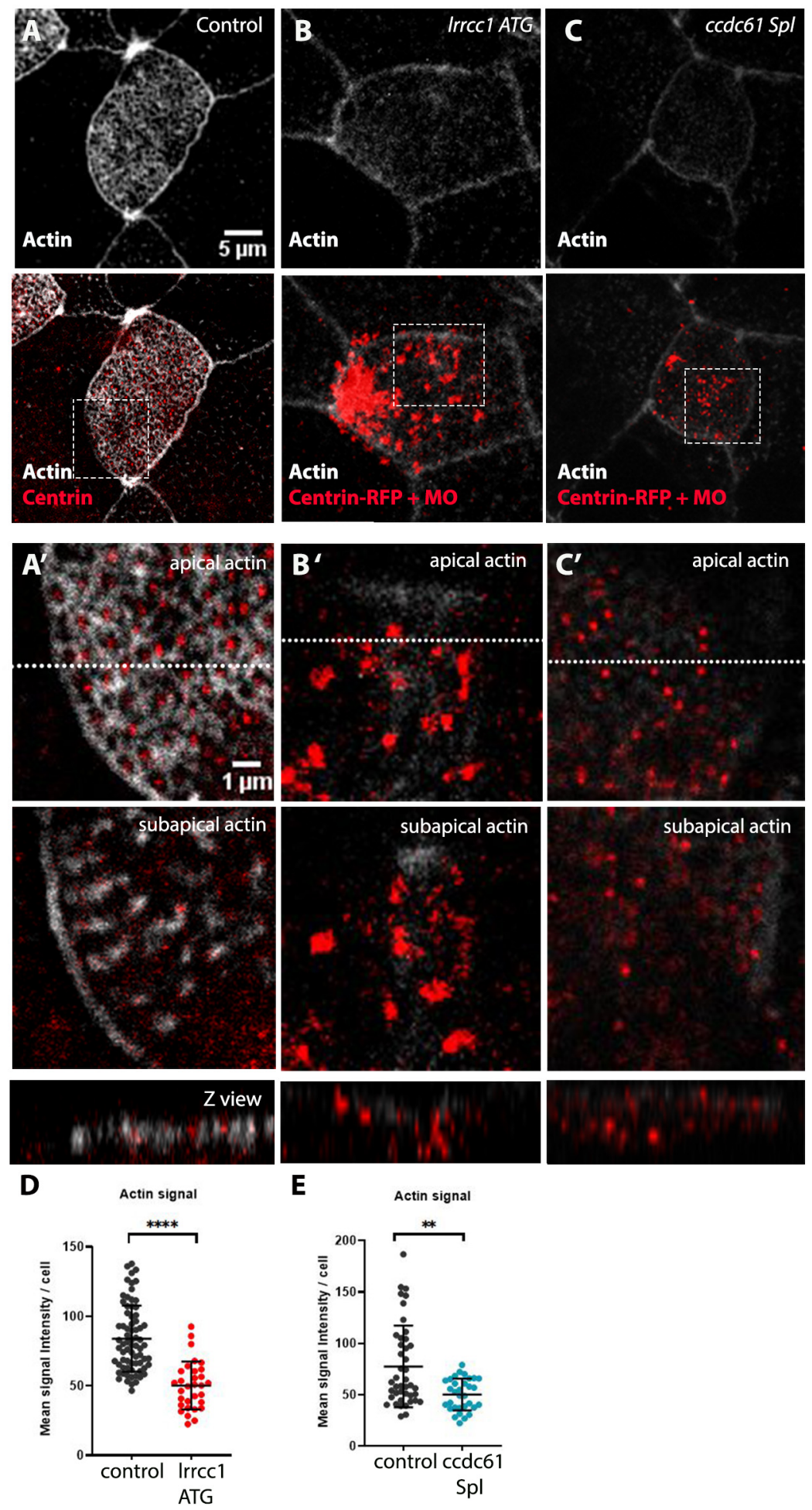


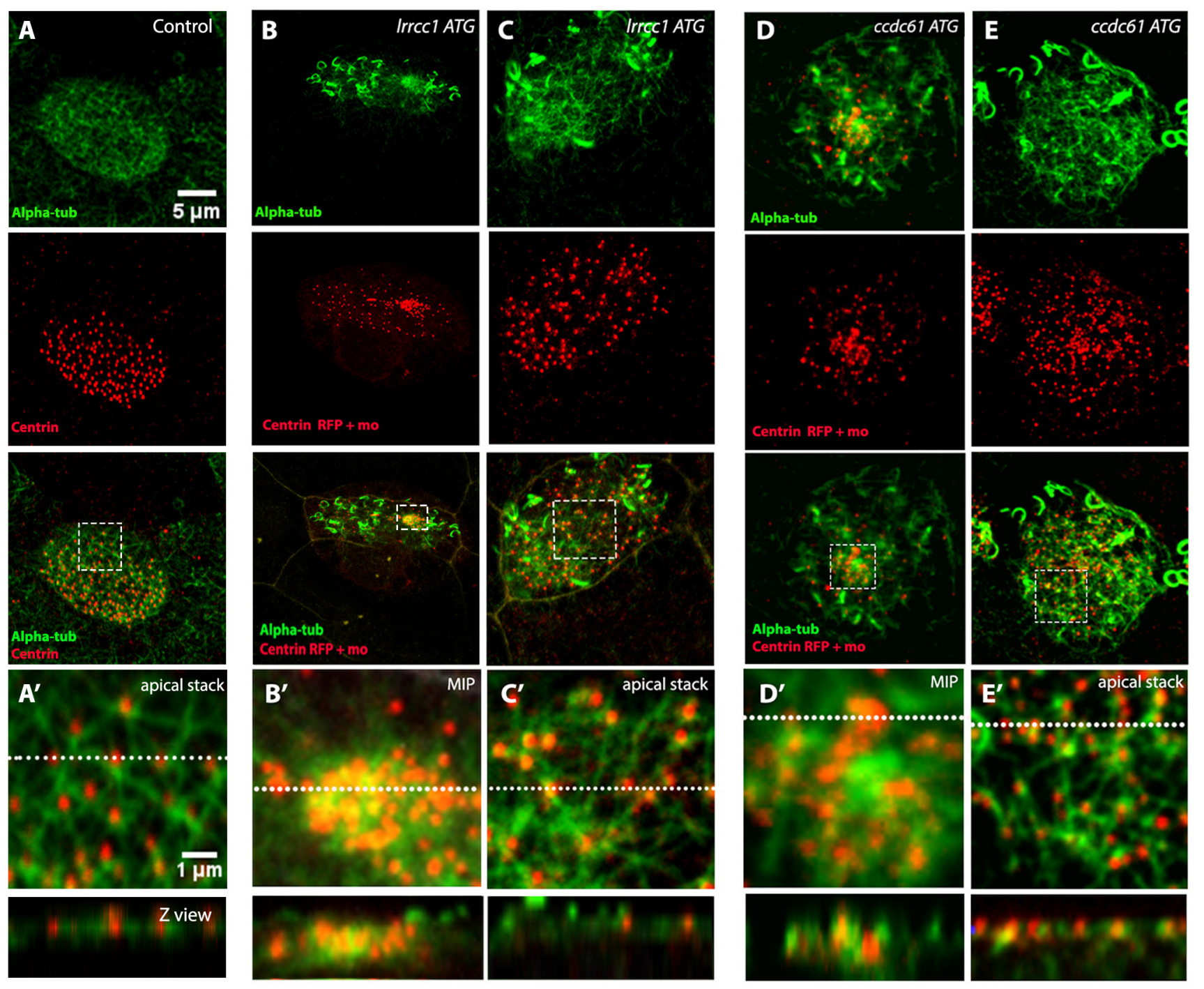




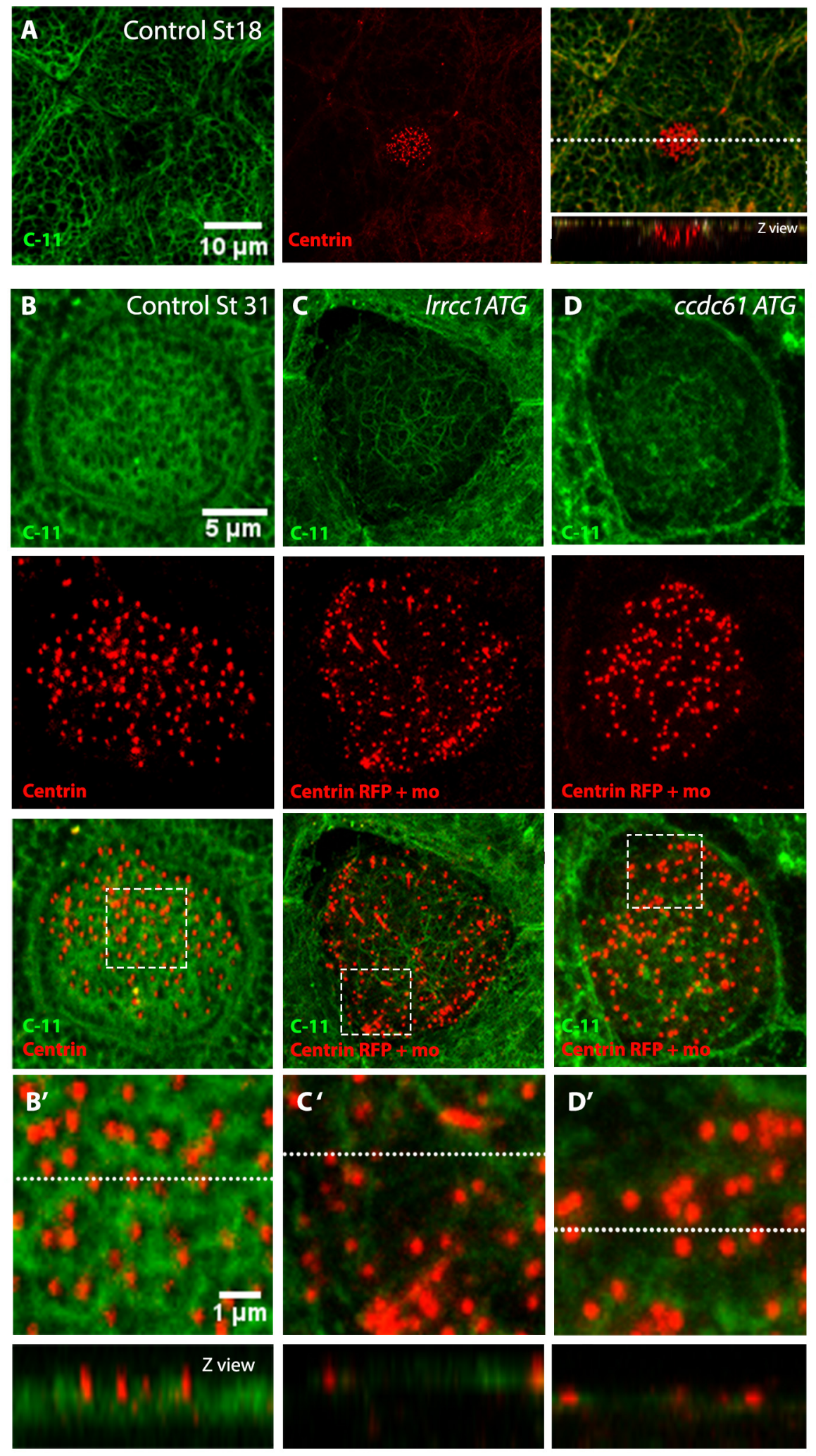


A Beating Frequency

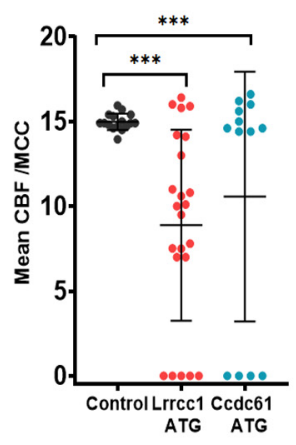

C

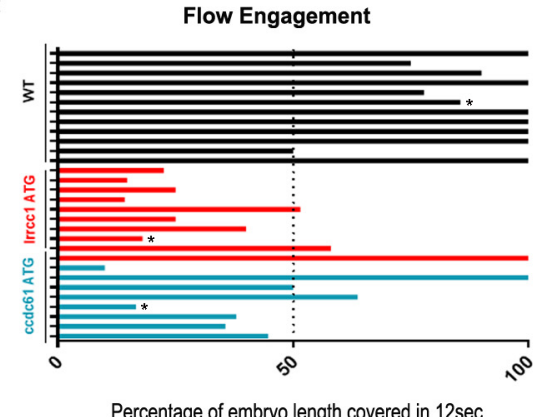

Percentage of embryo length covered in $12 \mathrm{sec}$

B

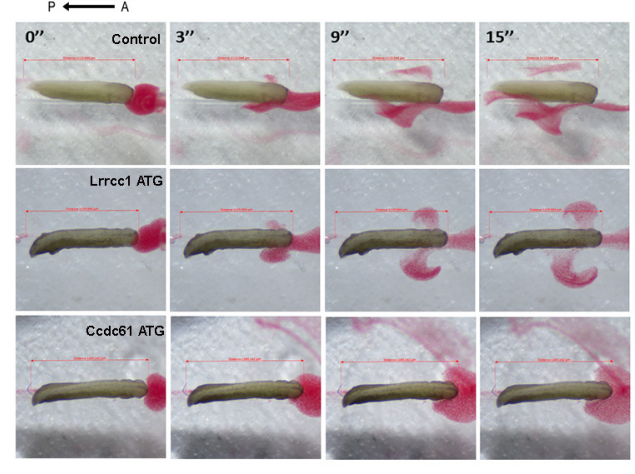

D Tadpole survival in presence of A.Hydrophila

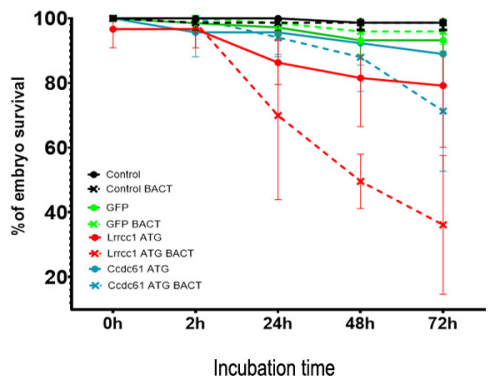



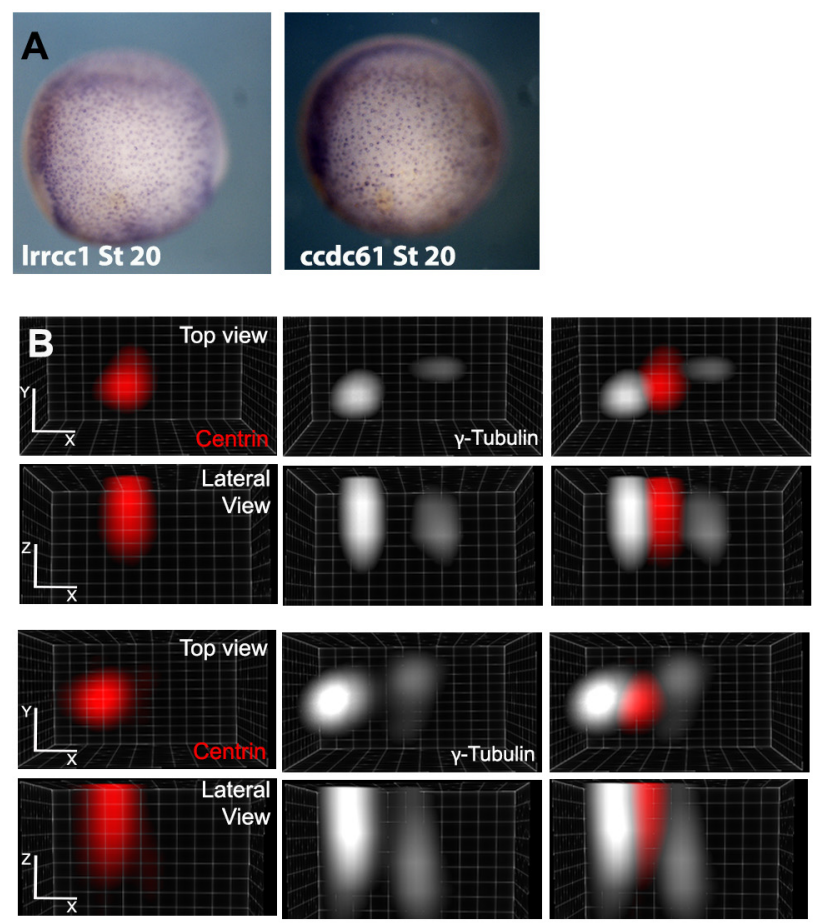
A

\section{Pre-mRNA}

\begin{tabular}{l} 
Ex1 1 In1 \\
MOATG \\
\hline MOSpI $\cdots$
\end{tabular}

Irrcc1.L

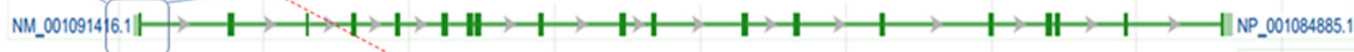

\begin{tabular}{l} 
Pre-mRNA \\
- Ex1 1 In 1 \\
\hline MoATG
\end{tabular}

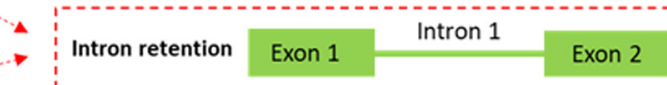

ccdc61.L

NM_001096129.11-

B

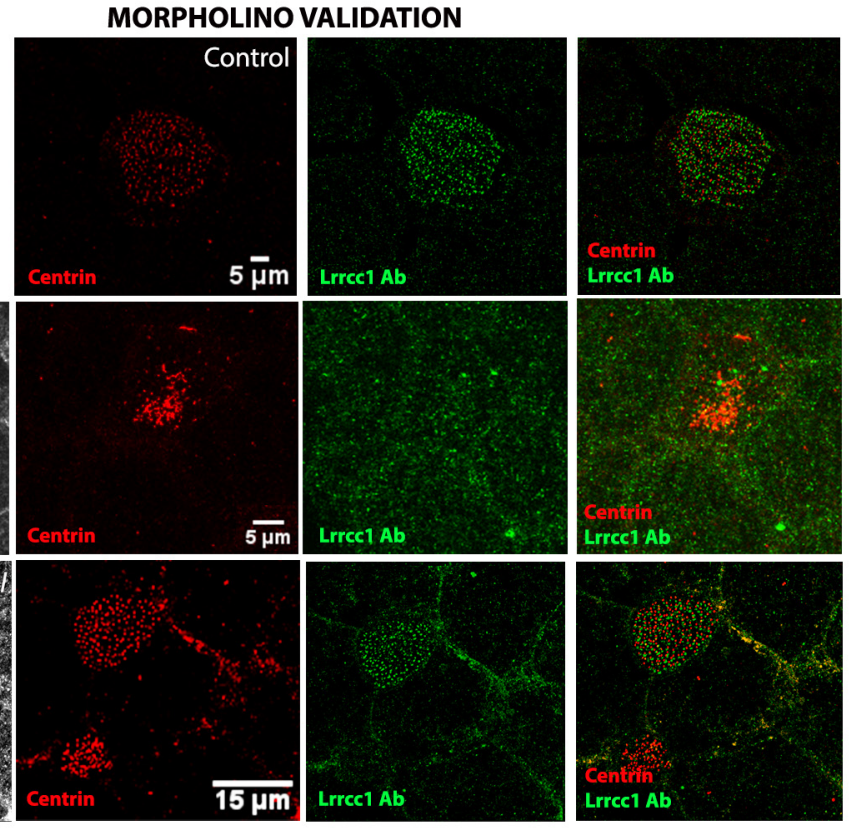

C

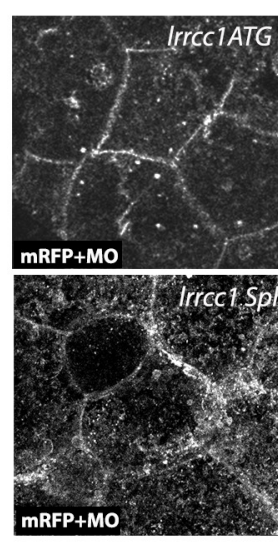

C
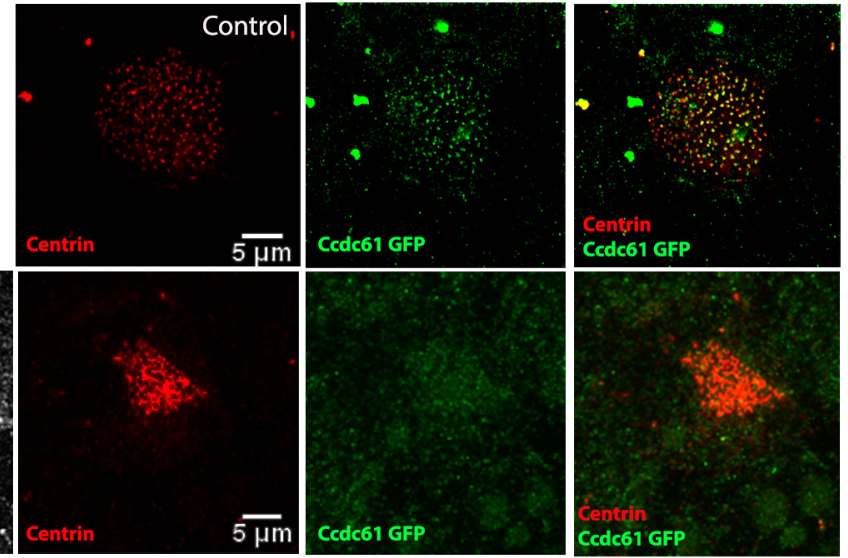


\section{A}

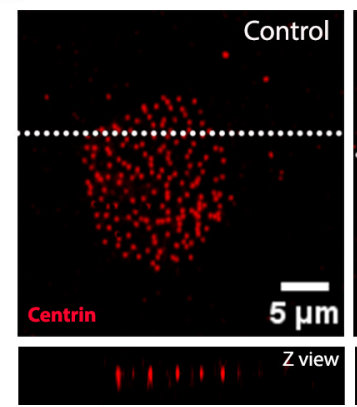

C
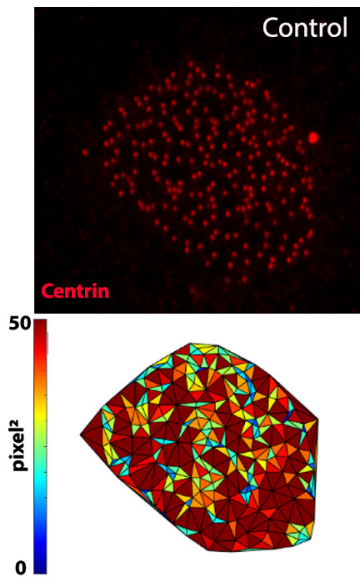

E
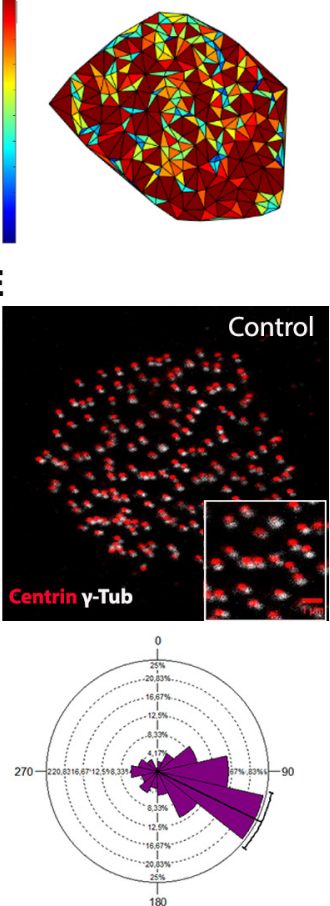

Basal Body DOCKING

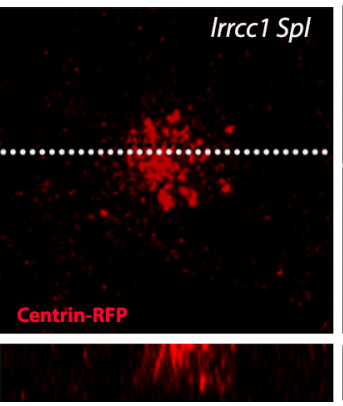

Basal Body SPACING
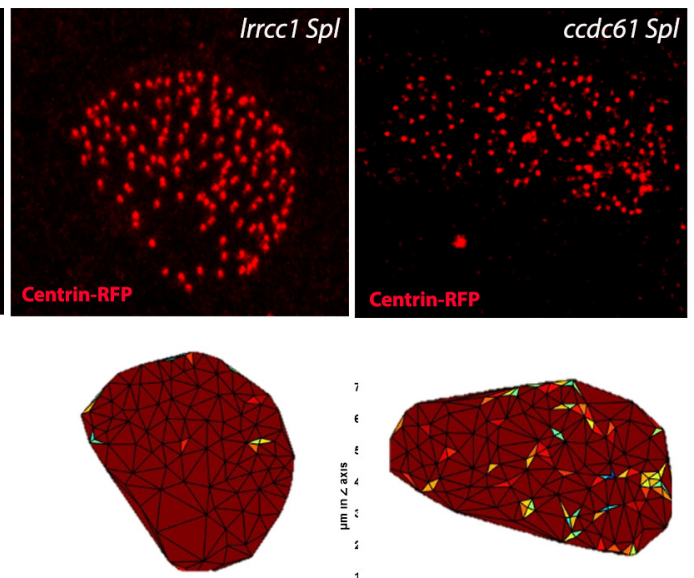

Basal Body POLARITY
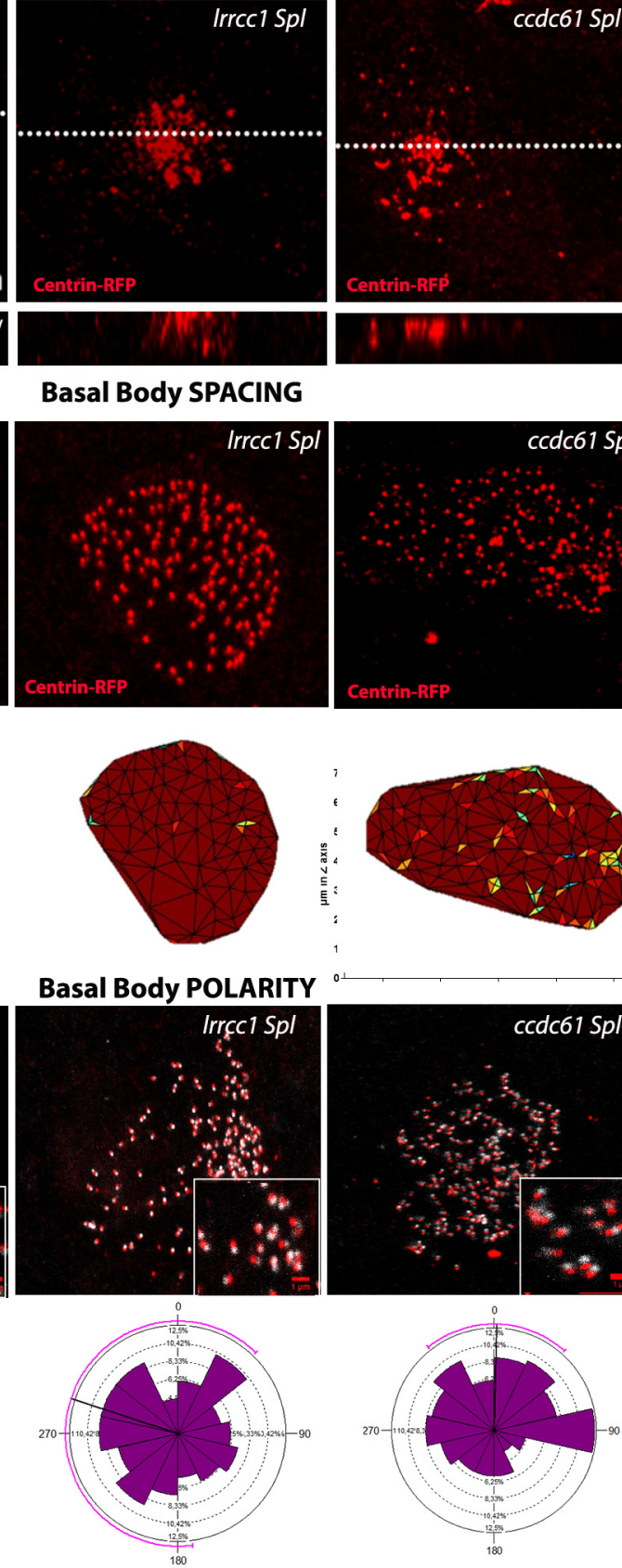

Sentrin-RFP
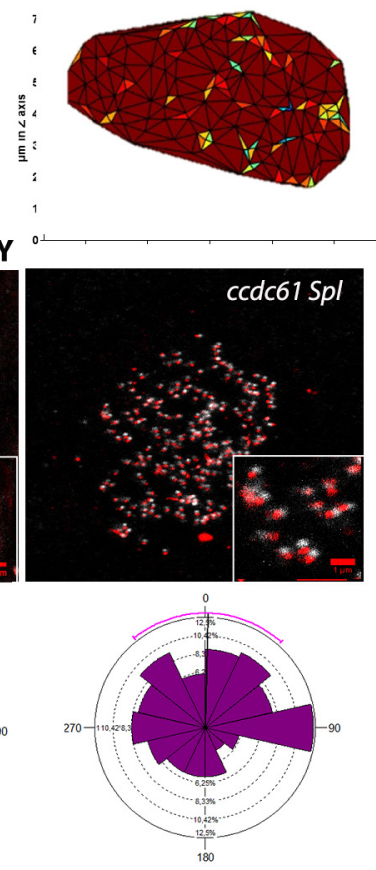

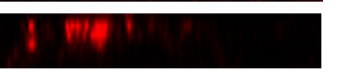

D

$\mathbf{F}$

每

Basal body polarization

G Non polarized MCCs
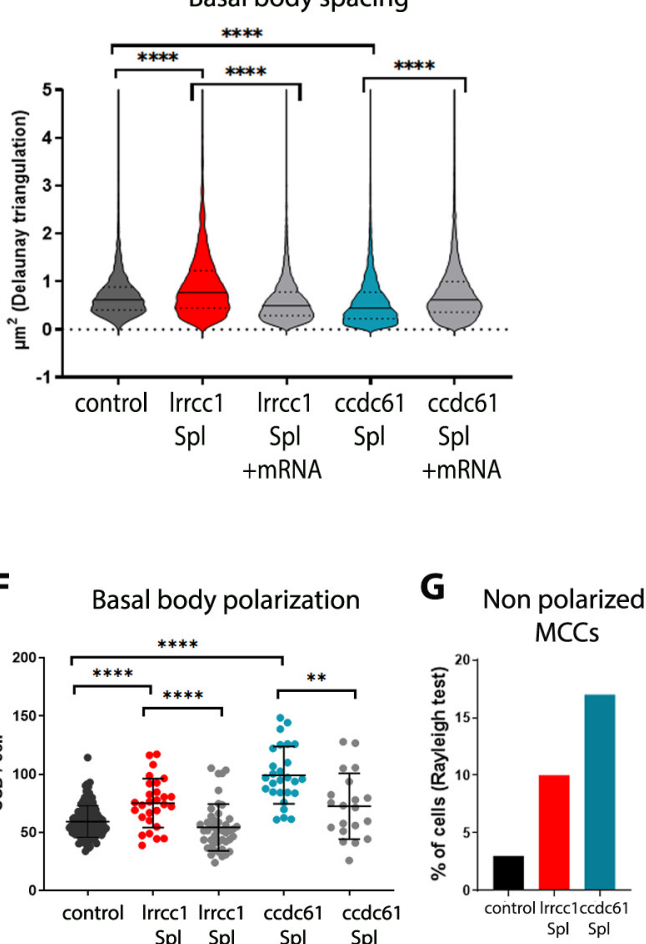

control Irrcc1 Irrcc1 ccdc61 ccdc6 Spl Spl Spl Spl +mRNA 

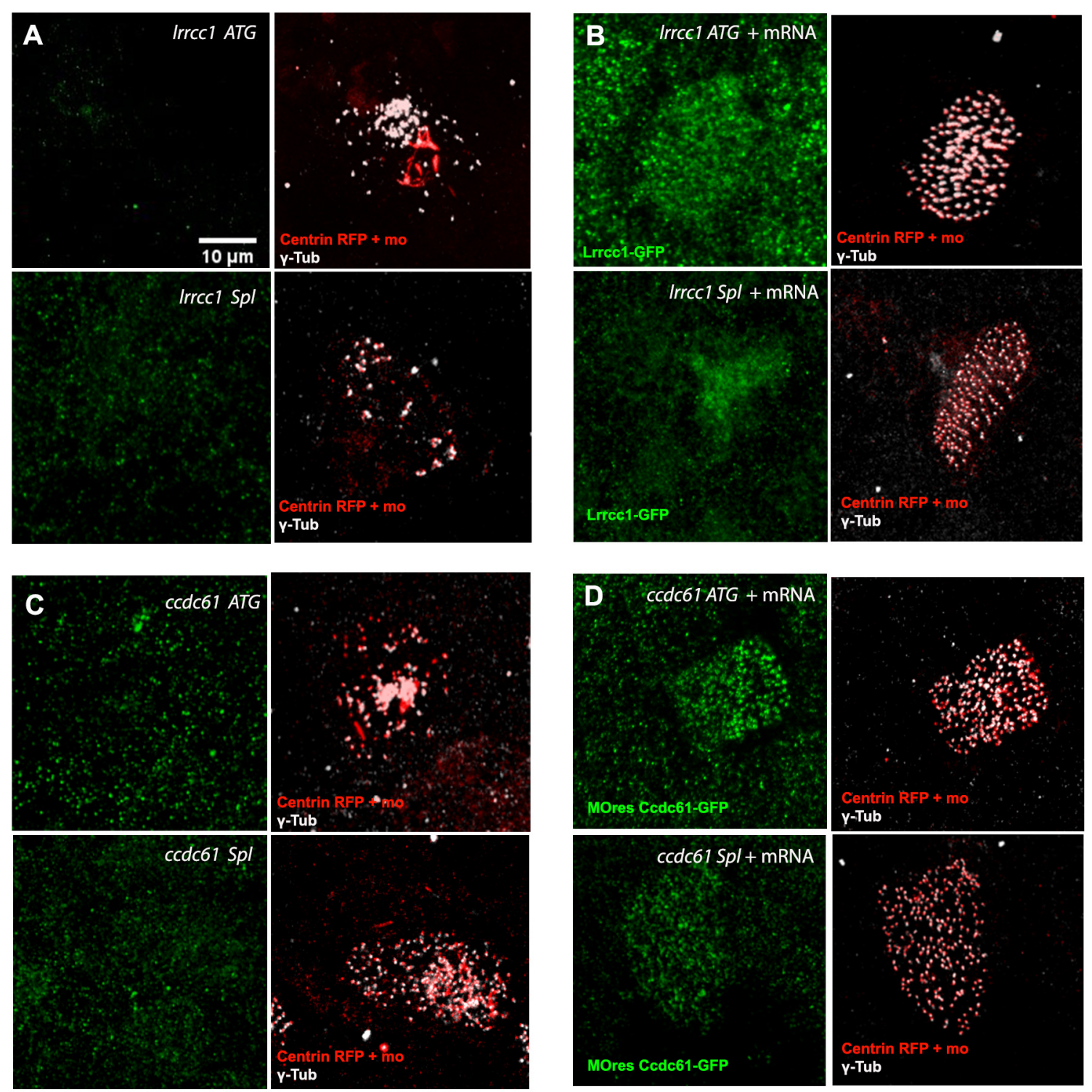


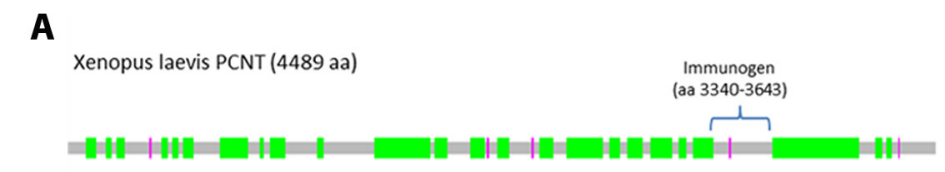

B

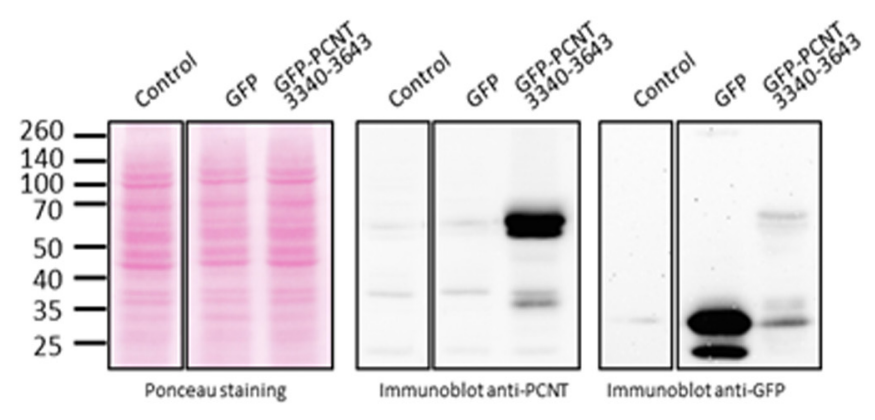

C

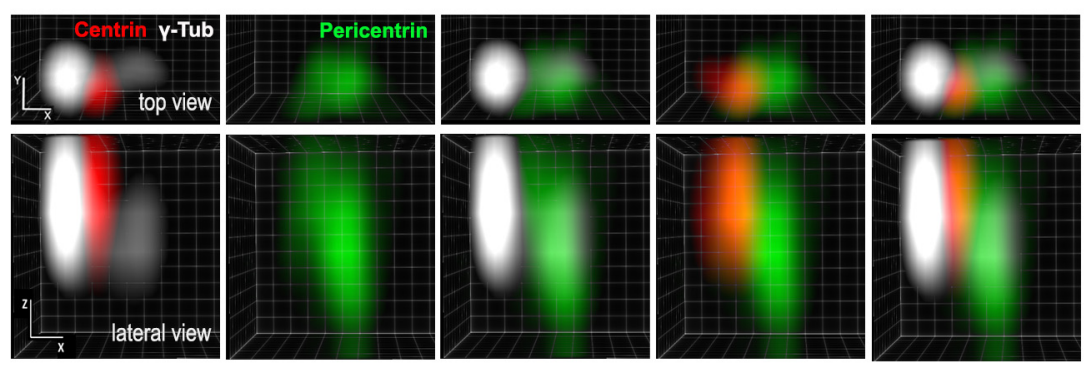




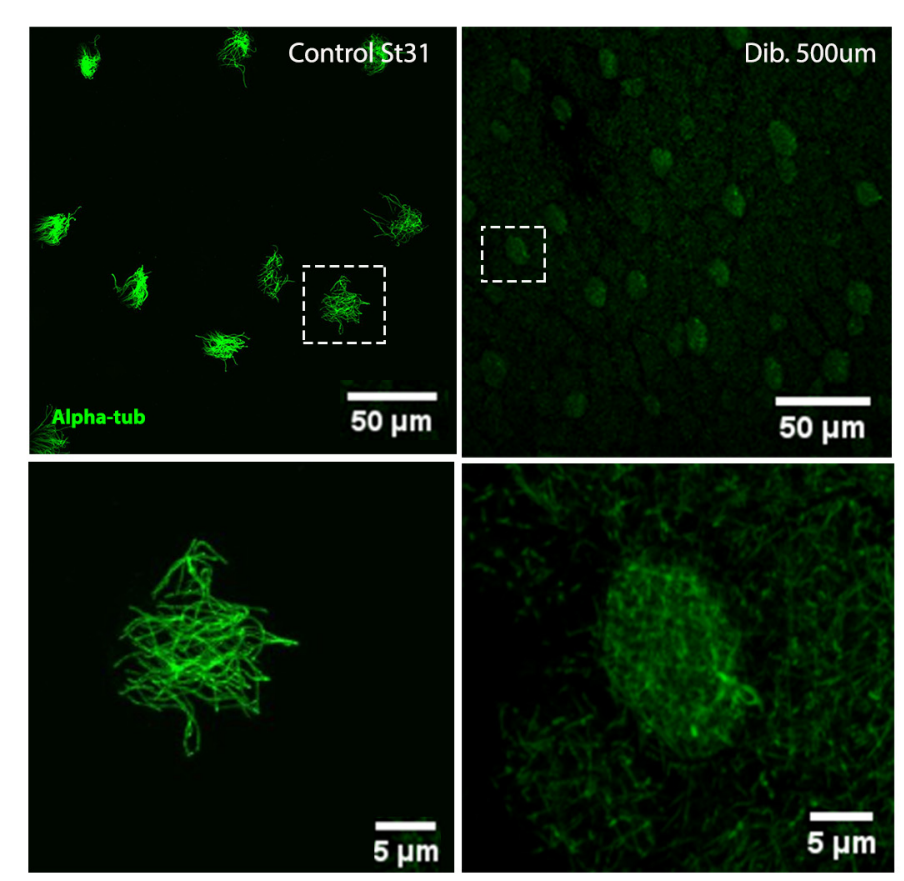



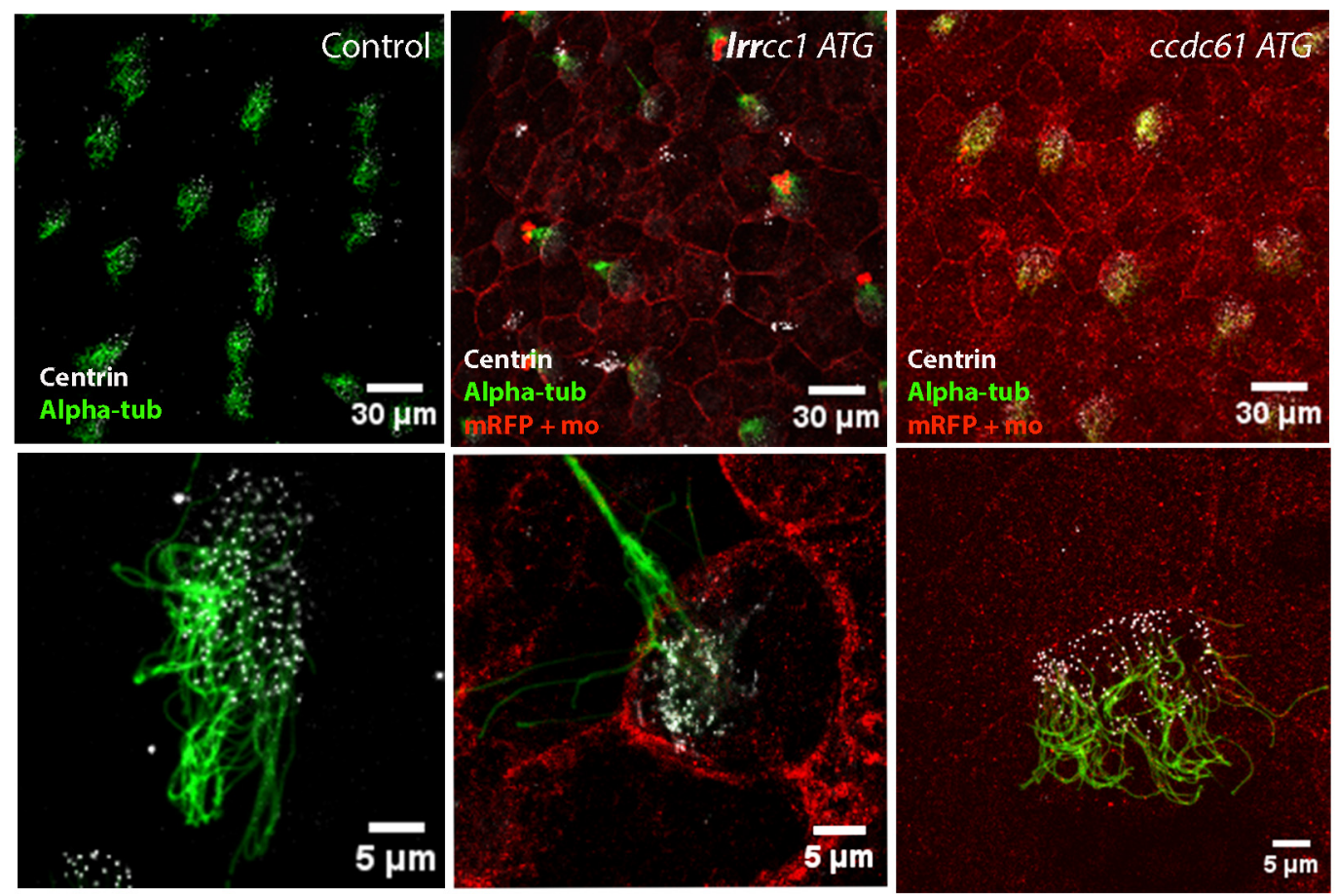